\title{
Searching for a solution to the cosmological constant problem - a toy model
}

\author{
Wojciech Tarkowski \\ St. Paul Research Laboratory, ul. Żegańska 24c/2, 04-713 Warsaw, Poland \\ e-mail: tarkowski@data.pl
}

\begin{abstract}
This paper concerns the so-called cosmological constant problem. In order to solve it, we propose a toy model providing an extension of the dimensionality of spacetime, with an additional spatial dimension which is macroscopically unobservable. The toy model introduces no corrections to most predictions of the "standard" general relativity regarding, among others, the so-called "five tests of general relativity". However, it seems that the toy model could provide an explanation to the flatness of circular velocity curves of spiral galaxies without introducing any dark matter. The proposed model has quite important cosmological consequences. By introducing certain corrections to Friedmann's currently accepted model(s), the toy model allows one to solve problems related to the present density of matter in the Universe and, finally, it does not contain the initial singularity.
\end{abstract}

PACS numbers: 04.20.-q, 04.20.Cv, 04.20.Jb, 04.90.+e, 98.62.Gq, 98.62.-g, 98.80.Es, $98.80 .+\mathrm{k}$ 


\section{Introduction}

There has probably never existed such a difference between predictions given by two commonly approved and powerful theories. On the one hand, astronomical observations place strong limits on the value of the cosmological constant $\lambda$ requiring it should not be greater than $10^{-52} \mathrm{~m}^{-2}$; see Ref. [1. On the other hand, the quantum theory predicts that anything contributing to the vacuum-energy density should act like a cosmological constant. Theoretical expectations thus give $\lambda$ of the order of $10^{70} \mathrm{~m}^{-2}$, which exceeds observational limits by about 120 orders of magnitude. This huge discrepancy is at the origin of a dilemma often referred to as the cosmological constant problem. Recently, many attempts have been undertaken to solve this paradox; see Ref. [2]. In this paper, we propose a new and very simple solution to it, which will be called a toy model.

\section{Einstein generalized equation}

We start this work by writing the Einstein generalized field equation,

$$
R_{\mu \nu}-\frac{1}{2} g_{\mu \nu} R+\lambda g_{\mu \nu}=\kappa T_{\mu \nu}
$$

where $R_{\mu \nu}, g_{\mu \nu}$ and $T_{\mu \nu}$, with $0 \leq \mu, \nu \leq k \in \mathcal{N}$, are the components of the Ricci, metric and stress-energy tensors, respectively, $\lambda$ denotes the cosmological constant, $R \equiv R_{\mu \nu} g^{\mu \nu}$, and $\kappa=-8 \pi G / c^{4}$; we also assume that the relationship between the Ricci and Riemann-Christoffel tensors is of the form $R_{\mu \nu} \equiv R_{\mu \nu \alpha}^{\alpha}$. Note that in our approach we consider the term $\lambda g_{\mu \nu}$ in Eq. (11) to be related to the left-hand side, i.e. the geometric side of the Einstein equation. Therefore, we believe that this term does not contribute to the stress-energy tensor $T_{\mu \nu}$.

It is clear that, assuming the value of the constant $\lambda$ to be of the order of $10^{70} \mathrm{~m}^{-2}$ in a four-dimensional $(1+3)$ spacetime, we are not able to obtain

any reasonable, i.e. consistent with observations, solution of Eq. (11). One of the possible ways out of this situation is to increase the spacetime dimensionality. Yet, how many additional dimensions are needed in such a case? We will answer this question by defining the vacuum stress-energy tensor $T_{\mu \nu}$ which has to render appropriate - from the point of view of the quantum field theory - parameters of the vacuum, i.e. among others its enormously 
large density of energy. To do this, let us appeal to the Newtonian gravity theory, which remains an excellent approach to Einstein's general relativity at relatively small distances and velocities, ${ }^{1}$ and note that - according to experimental data - the generalized Poisson equation [1] for the Newtonian gravitational potential $\psi$ in a small region of an empty space should take the form $\nabla_{\mathbf{r}}^{2} \psi \approx 0$ where $\mathbf{r} \equiv[x, y, z]$. Since for the generalized Poisson equation one has $\nabla_{\mathbf{r}}^{2} \psi \propto \operatorname{Tr}\left(\operatorname{diag} T_{\mu \nu}\right)$ so, consequently, $\operatorname{Tr}\left(\operatorname{diag} T_{\mu \nu}\right) \approx 0$ must occur, which - due to the large value of the constant $\lambda$ - obviously rules out simple solutions of the type of $T_{\mu \nu}= \pm \lambda g_{\mu \nu} / \kappa$ for all $\mu$ and $\nu$. At the same time we require space with the three spatial dimensions - the only ones which are subject to our direct perception - to be homogeneous, isotropic, and finally, to show a sufficiently small curvature for relatively short distances. Moreover, the additional spatial dimension(s) should not be directly observable. It is easy to demonstrate that the stress-energy tensor complying with all the above requirements should, after diagonalization, take the form $\left(\operatorname{diag} T_{\mu \nu}\right)=(X,-X, 0,0,0)$ - with an appropriately adjusted value of the quantity $X \in \mathcal{R}$ - which can be written as $\left(\operatorname{diag} T_{\mu \nu}\right)=\left(u_{v a c}, \widetilde{p}_{v a c}, 0,0,0\right)$, where we define the quantity $u_{v a c}$ as a vacuum-energy density and the quantity $\widetilde{p}_{v a c}-$ as a vacuum pressure. Then, the only non-vanishing components of the stress-energy tensor $T_{\mu \nu}$ are

$$
T_{\mu \nu}=\frac{\lambda}{\kappa} g_{\mu \nu} \quad \text { for } \quad \mu=0,1 \quad \& \quad \nu=0,1 .
$$

Hence, the spacetime of our toy model is five-dimensional $(1+4)$, of the form $\mathcal{R}^{1}$ (time) $\times \mathcal{R}^{1}$ (extra spatial dimension $) \times \mathcal{R}^{3}$ (three-dimensional space). For this and subsequent sections of this paper, the signature of the metric tensor is assumed to be equal to -3 , so the elements of the diagonalized metric tensor have the signs $(+,-,-,-,-)$. We also assume that the cosmological constant has a negative value, and that $|\lambda| \sim 10^{70} \mathrm{~m}^{-2}$. Consequently, the value of the vacuum-energy density

$$
u_{v a c}=-\frac{c^{4} \lambda}{8 \pi G},
$$

where $G$ denotes the Newtonian gravitational constant and $c$ stands for the speed of light, is of the same order of magnitude as that predicted by the

\footnotetext{
${ }^{1}$ Note, however, that the Newtonian theory remains valid also while describing the relatively slow mean motion of a relativistic fluid, i.e. the fluid whose (most) particles move with velocities which are comparable to the velocity of light.
} 
quantum theory. Note that $T_{\mu \nu}$, for $\mu=0,1$ and $\nu=0,1$, is the stressenergy tensor of an ideal fluid which satisfies the vacuum equation of state, $u_{v a c}=-\widetilde{p}_{v a c}$, and the (negative) vacuum pressure

$$
\widetilde{p}_{v a c}=\frac{c^{4} \lambda}{8 \pi G}
$$

comes exclusively from the additional spatial dimension. In other words, the pressure $\widetilde{p}_{v a c}$ acts along the extra spatial dimension (further denoted by $a$ ), i.e. orthogonally (on)to hypersurfaces with constant values of the coordinate a. Pressure along the three "macroscopic" spatial dimensions is equal to zero.

Note also that for Eq. (11) with the stress-energy tensor defined by expression (2), the corresponding generalized Poisson equation takes the form $\nabla_{\mathbf{r}}^{2} \psi=-\kappa c^{2}\left(u_{v a c}+\widetilde{p}_{v a c}\right) / 2=0$, according to our expectations and to the requirement imposed while constructing the stress-energy tensor $T_{\mu \nu}$ at the beginning of this section; see also section 5.1 of this paper.

It is worth emphasizing that the Einstein equation (10) with the stressenergy tensor given by expression (21) implies that the only non-vanishing components of the Ricci tensor $R_{\mu \nu}$ are

$$
R_{\mu \nu}=\lambda g_{\mu \nu} \quad \text { for } \quad \mu=0,1 \quad \& \quad \nu=0,1
$$

\section{Solution for an empty space}

The solution of Eq. (11) with the stress-energy tensor given by expression (2) reads

$$
d s^{2}=\left(1+|\lambda| a^{2}\right) c^{2} d t^{2}-\left(1+|\lambda| a^{2}\right)^{-1} d a^{2}-d x^{2}-d y^{2}-d z^{2}
$$

where $a \equiv x^{1}$ and $(x, y, z) \equiv\left(x^{2}, x^{3}, x^{4}\right)$, with $x^{1}$ and $\left(x^{2}, x^{3}, x^{4}\right)$ denoting the "micro" and macrospace coordinates, respectively. ${ }^{2}$

Let us first investigate the properties of the metric (6) on a "microscale". Taking $(x, y, z)=$ const we obtain

$$
d s^{2}=\left(1+|\lambda| a^{2}\right) c^{2} d t^{2}-\left(1+|\lambda| a^{2}\right)^{-1} d a^{2} .
$$

\footnotetext{
${ }^{2}$ The term "microspace" is used here to emphasize that the additional spatial dimension is macroscopically, or directly unobservable, which will be shown later.
} 
This metric describes the covering surface $\mathcal{R}^{1} \times \mathcal{R}^{1}$ of the anti-de Sitter two-dimensional spacetime with the negative cosmological constant $\lambda$. It is known in turn, that the anti-de Sitter spacetime of the form $\mathcal{S}^{1} \times \mathcal{R}^{1}$ has no Cauchy surfaces and contains "global" closed time-like curves [3, 4. Namely, after a (coordinate) time

$$
T_{l}=\frac{2 \pi}{c \sqrt{|\lambda|}}
$$

has elapsed, the observer located at any place where the condition $a=$ const is satisfied, would retrace his own life history (of course, the covering surface given by the metric (7) does not possess this property any more). Hence, one obtains a "natural" unit imposed on the anti-de Sitter spacetime: the coordinate time $T_{l} \sim 10^{-43} \mathrm{~s}$. Note that the maximum distance, which can be covered during the time $T_{l}$ by a signal or a particle, is equal to $L \equiv c T_{l} \sim 10^{-35} \mathrm{~m}$. In the next section it will be shown that no particle with a finite energy, moving in the spacetime described by expression (6), could irrevocably leave the nearest neighbourhood $a \sim \pm L$ of the macrospace given by $a=0$. First, however, we should point out that the quantity $T_{l}$ is of the order of the Planck time $T_{P l}=\left(G \hbar / c^{5}\right)^{1 / 2}$, where $\hbar \equiv h /(2 \pi)$ denotes the Planck constant, and $L$ is of the order of the Planck length $L_{P l}=\left(G \hbar / c^{3}\right)^{1 / 2}$. Bearing in mind the formula (8), one then can assume that in our toy model the value of the cosmological constant is given by the combination of the fundamental constants of nature,

$$
\lambda=-\frac{c^{3}}{G \hbar} \cong-3.829 \times 10^{69} \mathrm{~m}^{-2} .
$$

\subsection{Equations of motion}

Now we will present considerations which lead to the conclusion that the additional spatial dimension is directly unobservable. Taking the metric (6) we obtain integrated equations of the motion for a massless as well as an ordinary (i.e. possessing a finite mass) test particle ${ }^{3}$ in the absence of any potentials,

$$
\left(\frac{d a}{d \gamma}\right)^{2}=U^{2}-\left(1+|\lambda| a^{2}\right)|\mathbf{p}|^{2}
$$

\footnotetext{
${ }^{3}$ Note that in this paper we investigate the behaviour of uncharged particles only.
} 


$$
m^{2}\left(\frac{d a}{d \sigma}\right)^{2}=U^{2}-\left(1+|\lambda| a^{2}\right)\left(m^{2}+|\mathbf{p}|^{2}\right)
$$

respectively. As usual, the quantities $\gamma$ and $\sigma$ denote here affine parameters; in particular, the parameter $\sigma$ may be equal to the proper time $\tau$ of a particle with a finite mass. The constant quantities $m, U$ and $\mathbf{p}$ are the particle's rest-mass, total energy and three-dimensional momentum vector in the "macroscopic" space $a=0$, respectively, and $c$ denotes the velocity of a massless particle with regard to the hypersurface $a=0$. Finally, $U \equiv p_{0}$, $|\mathbf{p}|^{2}=p^{2} \equiv-\sum_{i=2}^{4} p_{i} p^{i}=\sum_{i=2}^{4}\left(p^{i}\right)^{2}$, and $p^{a} \equiv p^{1}$ is equal to the square root of the left-hand side of Eqs. (10) or (111). It is then easy to conclude that only particles with huge energies $U$ are able to surmount the incredibly deep potential well ${ }^{4}$ - proportional to $\left(1+|\lambda| a^{2}\right)^{1 / 2}$, and penetrate the additional spatial dimension $a$ on a scale much larger than $L \sim|\lambda|^{-1 / 2} \cdot\left({ }^{5}\right)$ Moreover, there exists only a single stable stationary point for the particle moving in the additional spatial dimension, given by

$$
0=\frac{d}{d a} \sqrt{1+|\lambda| a^{2}}
$$

it is of course $a=0$, i.e. the "ordinary" four-dimensional "macroscopic" spacetime. The acceleration of any object moving towards this point (or, to be more precise, this hyperspace) would be enormously large even for extremely short distances $a$.

Assuming the right-hand sides of Eqs. (10) and (11) to be non-negative, one may solve both of them, obtaining

$$
\begin{aligned}
& a(\gamma)= \pm \sqrt{\frac{U^{2}-p^{2}}{p^{2}|\lambda|} \sin (p \sqrt{|\lambda|} \gamma)} \\
& a(\eta)= \pm \sqrt{\frac{U^{2}-\left(m^{2}+p^{2}\right)}{\left(m^{2}+p^{2}\right)|\lambda|}} \sin \left[\sqrt{\left(m^{2}+p^{2}\right)|\lambda|} \eta\right]
\end{aligned}
$$

${ }^{4}$ Of course, this is true only for particles which fulfil the condition $p \neq 0$ or $m \neq 0$. Otherwise, i.e. if $p=0$ and $m=0$, one has $a \rightarrow \pm \infty$ even for an arbitrarily small (but non-zero) value of the energy $U$; see Eqs. (10) and/or (11).

${ }^{5}$ See also Ref. [5]. The authors conclude that the extra dimension(s), even though non-compactified, would be unobservable directly, if ordinary "three-dimensional" (light) particles were confined inside a potential well which is narrow enough along the additional spatial dimension(s), but flat along the three physical ones. As we have seen, for the spacetime with the metric (6), the two above requirements are satisfied. 
the quantity $\eta \equiv \sigma / m$ in expression (14) is the affine parameter.

First let us focus on the formula (13) . Comparing $p[G|\lambda| /(\hbar c)]^{1 / 2}=\omega$ for the argument of the sine function (the momentum $p \equiv|\mathbf{p}|$ is expressed here in SI units) and substituting $\lambda=-c^{3} /(G \hbar)$, we arrive at the fundamental quantum relation between the momentum and the wave frequency, $p=\hbar \omega / c$. However, this formula now has a completely new meaning: the "ordinary" three-dimensional momentum of a massless particle is proportional to the frequency of its oscillations in the additional spatial dimension. It should be pointed out that this relationship results exclusively from the spacetime geometry.

Now let us define $E$ conventionally as the energy of a particle in the four-dimensional special-relativistic spacetime $a=0$ in the absence of the additional spatial dimension; it will further be demonstrated that in the toy model, for the case of a particle or of a system of particles moving along the geodesic line(s), this quantity remains quasi-constant for sufficiently long time intervals. Since $E \equiv c p$, we arrive at the familiar expression called the Planck formula, $E=\hbar \omega$. Similarly, in the case of expression (14), we have $E^{2} \equiv m^{2} c^{4}+c^{2} p^{2}=\hbar^{2} \omega^{2}$. For the four-dimensional coordinate system in the spacetime given by $a=0$, in which the particle rests $(p=0)$, we may then write $E_{0}=m c^{2}=\hbar \omega_{0}$, where $\omega_{0}$ is the oscillation (or wave) frequency of the particle in this system. The formula

$$
E^{2}-c^{2} \mathbf{p}^{2}=m^{2} c^{4}=\hbar^{2} \omega_{0}^{2}=\hbar^{2} \omega^{2}-\hbar^{2} c^{2} \mathbf{k}^{2},
$$

where $\mathbf{k}$ denotes the three-dimensional wave-vector in the "macroscopic" space $a=0$, is satisfied in another coordinate system in which the particle moves. Here, for a moment, we made use of Einstein's special relativity theory which corresponds to the spacetime determined by $a=0$ in the metric (6). Special relativity explicitly and unambiguously determines the extension of the Planck formula $E=\hbar \omega$ on four-dimensional vectors present in this theory. Namely, in special relativity both the structures: $(E / c, \mathbf{p})$ and $(\omega / c, \mathbf{k})$ form the four-vectors. The energy $E$ in the four-vector is accompanied by the three momentum components while the wave frequency $\omega$ goes with the three components of the wave-vector $\mathbf{k}$. The formula (15) reflects the fact that both the four-vectors have constant lengths in different coordinate systems of the spacetime $a=0$. The particle's momentum vector $\mathbf{p}$ is in the direction of the oscillation, or wave normal, so it has the same direction as the wave-vector $\mathbf{k}$; thus, comparing $E=\hbar \omega$ in Eq. (15) we arrive at the de Broglie relation $\mathbf{p}=\hbar \mathbf{k}$. 
Let us note that the trajectory of a particle is, in fact, identical to the trajectory of a certain (uniform) transverse plane wave which propagates in the "macroscopic" three-dimensional space $a=0$; see the formulae (13) and/or (14). In other words, one can regard the particle's trajectory as a trajectory of a certain wave. In turn, we know that the simplest plane wave is characterized by the wave frequency $\omega$ and by the wave-vector $\mathbf{k}$ that has the direction coincident with the direction of the wave propagation and the length $2 \pi / \lambda$, where $\lambda$ denotes the wavelength. Such a wave may be described by the following representation

$$
B \exp [i( \pm \mathbf{k} \cdot \mathbf{r}-\omega t)]
$$

where $B$ denotes the wave amplitude, $i$ is the imaginary unit, and $\mathbf{r} \equiv$ $\left[x^{2}, x^{3}, x^{4}\right]=\left[x_{2}, x_{3}, x_{4}\right]$. On the other hand, for the discussed particle we have $\omega=E / \hbar$ and $\mathbf{k}=\mathbf{p} / \hbar$. Hence, the simplest wave representation of any $\left(\right.$ free $\left.^{6}\right)$ particle can be written as

$$
B \exp \left[\frac{i}{\hbar}( \pm \mathbf{p} \cdot \mathbf{r}-E t)\right] \equiv D(\mathbf{r}) \exp \left(-\frac{i}{\hbar} E t\right)
$$

where the function $D(\mathbf{r})$ denotes the spatial part of the wave representation. Note that the complex time factor $\exp (-i E t / \hbar) \equiv \exp (-i \omega t)$ or the linear superposition of such factors with different oscillation frequencies $\omega$, present in the above representation and in most wave-functions of quantum mechanics (i.e. for the cases of time-independent external fields, or stationary states), obtains simple interpretation in the toy model. It just corresponds to the oscillations of the investigated object in the additional spatial dimension.

Let us proceed with the analysis of the relationships (13) and (14). In particular, assuming that $E=\hbar \omega=0$, from the formulae (13) or (14) we obtain $a= \pm U \gamma$ or $a= \pm U \eta$, respectively. This means that particles satisfying the conditions $E=0$ (or $\omega=0$ ) and $U \neq 0$ can leave the spacetime $a=0$ irrevocably. Objects with the "three-dimensional" energies $E$ being - instead of real numbers - imaginary ones, behave in a similar way. Note that the above condition does not have to be fulfilled by the representation (17). Depending on the sign of the imaginary component of the quantity $E$, it could either approach zero or amplify illimitably with the lapse of time $t$. Such a behaviour of the representation (17) is not surprising, as the equations

\footnotetext{
${ }^{6}$ Note that expression (6) is actually a generalized special-relativity metric, since it incorporates no gravitational effects (from masses) or other interactions.
} 
of quantum mechanics do not take into account the existence of objects with complex energies $E$. Thus, the above discussion clearly corresponds to the $a$ priori requirement of quantum mechanics that demands the Hamiltonian of an object or, more generally, of a quantum system to be a Hermitian operator, i.e. to have eigenvalues in real numbers, since those eigenvalues correspond to real, i.e. measurable physical quantites. ${ }^{7}$ And indeed, according to the toy model, the lack of fulfillment of this requirement - which means that the energy $E$ of an object has not a real value - results in the escape of a given object from the space $a=0$. For instance, such a situation could occur for an "exotic" kind of particles which are tachyons. Provided that the tachyonic energy $E$ fulfils the condition $E^{2}<0$, we then have $\omega^{2}<0$, so for the values of $U$ such that $U^{2}>E^{2}$ one obtains $a(\eta) \sim \pm \sinh (|\omega| \eta)$ and the tachyon would disappear in the additional spatial dimension $a$.

Now let us consider the equality (14). Assuming that $p=0$ and hence $E=m c^{2}=\hbar \omega$, we obtain a formula describing the motion of a particle that appears in the "observable" zone $a \in[-L, L]$ periodically but each time for a very short, comparable to $T_{l}$, time only. Such particles may correspond in our toy model to the actual phenomenon of the zero-point energy. Note that these particles behave exactly in the way the Planck oscillators do. We can also state that the toy model permits one to pose the rarely asked question beginning with the word why? Namely why, in - or with respect to - any (inertial) coordinate system in the spacetime $a=0$, massless particles have always the same velocity, equal to the speed of light $c$ ? The toy model provides an opportunity to answer this question. Let us imagine the massless particle, whose velocity is not constant in every coordinate system of the spacetime $a=0$. Then one can find such a coordinate system in which the particle velocity is equal to zero in the three-dimensional macrospace $a=0$, so the particle seems to remain motionless if only the macrospace $a=0$ is taken into account. However, in this coordinate system one has $a= \pm U \gamma$, and in effect the particle leaves the spacetime $a=0$ irrevocably, never crossing it again. In another coordinate system of the spacetime $a=0$, in which the particle is moving, we obtain $a \sim \pm \sin (\omega \gamma)$. In such a case, the particle remains in the "neighbourhood" of the spacetime $a=0$, perpetually crossing the hypersurface $a=0$. Thus we arrive at the evident contradiction, as in one coordinate system of the spacetime $a=0$ the number of crossing

\footnotetext{
${ }^{7}$ Note, however, that for instance the so-called quasi-stationary states are formally described in quantum mechanics with the use of the complex energy $E$, whose imaginary part has a negative value.
} 
of the hypersurface $a=0$ is equal to zero, whereas in another system it equals a certain natural number which grows with increasing the parameter $\gamma$. Thus, we reach the obvious conclusion that the constant velocity of massless particles in all coordinate systems of the spacetime $a=0$ ensures the selfconsistency of the toy model.

It is also worth mentioning another important feature of the solutions (13) and (14). Namely, according to Eqs. (10) and (11), the arguments of the sine functions in these expressions can have a minus sign, corresponding to particles with negative energies $E$, i.e. antiparticles. They would then be formally equivalent - if taking into account their motion with respect to the dimension $a$ - to the "ordinary" particles with positive energies $E$, but with the symmetrically reversed $(\eta \rightarrow-\eta)$ lapse of the affine parameter, or the proper time, $\gamma$ or $\eta$. Taking into account other, "macroscopic" spatial dimensions $x, y$ and $z$, the antiparticle would then be nothing but the particle moving - with respect to the spacetime $a=0$ - in the opposite direction to that of the motion of an "ordinary" particle. Note as well that, according to the formulae (13) and/or (14) and to the relationship $\sin (-x)=-\sin x$, the antiparticle would also be formally equivalent to the "ordinary" particle with the same, as for the antiparticle, lapse of the affine parameter $\gamma$ or $\eta$, but moving in the opposite direction (only) with respect to the dimension $a$.

Now we will draw some attention to the quantity $U$. It is the total energy of a particle, which remains constant for particles travelling along the geodesic line, since the metric (6) is independent of time. A condition, which should be satisfied by the energy $U$ if the particle's trajectory is to be time-like or null (as calculated with respect to the dimension $a$ ), can easily be derived,

$$
U^{2} \leq \hbar^{2}|\lambda| c^{2}+E^{2}
$$

Hence, the maximum depth the particle can penetrate into the additional spatial dimension is equal to

$$
a_{\max }= \pm \frac{\hbar c}{E}
$$

In particular, we obtain $a_{\max } \rightarrow \pm \infty$ for $E \rightarrow 0$.

So far, within the framework of our model, it is difficult to add anything concerning the quantity $U$. Let us imagine, however, a particle with a total energy $U$, an energy $E$ in the three-dimensional space $a=0$, and a frequency $\omega$. The particle moves with a uniform rectilinear motion with respect to 
the spacetime $a=0$ and simultaneously oscillates in the additional spatial dimension $a$. Then, let us assume that this particle encounters a potential barrier $V>E$ on its way; the potential is relatively shallow with respect to the additional spatial dimension; see Fig. 1. It is evident that an ordinary "classical" particle, moving only in the four-dimensional spacetime $a=0$, is not able to surmount the potential barrier higher than the particle's total energy $E$. When an additional spatial dimension is present, however, then the situation changes substantially. If the potential $V$ is very shallow with respect to the extra spatial dimension, then the particle can circumvent it (see Fig. 1), even when its total energy $U$ does not exceed $E$ by much. There is nothing puzzling about this phenomenon; on the contrary, its simplicity - which is the result of purely geometric relationships between the particle's motion and the spacetime configuration - is striking. The particle vanishes from "our" spacetime, given by $a=0$, just in front of the barrier to reappear soon after circumventing it. There is obviously a certain possibility that the particle might be reflected from the barrier. It is clear that the transmission (or reflection) coefficient depends on the particle's energy $E$, so - consequently - on its oscillation frequency $\omega$ as well as on parameters characterizing the potential barrier. In such a case the "hidden" (but inherent) parameter $U$ can also be a quantity that significantly influences the particle's behaviour. ${ }^{8}$ It is difficult to say what laws govern this parameter in a multi-particle system: is it characterized by some probability distribution, in the common sense of this notion coming from statistical physics? If it is, what does such a probability distribution depend on? One expects that in the case of more "realistic" particles than those just discussed (i.e. possessing spin, charge, etc.), in their description there would also appear "hidden" parameters other than $U$, characterizing for instance the total four-dimensional spin and/or charge of the object, or of the system of objects. It is possible that "hidden" parameters may be responsible for subtle correlations between distant objects which were previously interacting.

Note that the fact of the existence of the "hidden" parameters - such as the energy $U$, or the four-dimensional "super-spin" and/or "super-charge" of the system - indicates that the toy model is a non-local theory which contains hidden variables. Thus, the toy model - fulfilling the Bell inequality

\footnotetext{
${ }^{8}$ On the other hand, let us note that - similarly as in the "standard" quantum mechanics - many of the measurable quantities associated with particles (or waves), according to the formulae (13) and (14), seem to be independent of some of the particle/wave parameters, such as (for instance) its oscillation amplitude, and hence - also of the energy $U$.
} 
- simultaneously could reconcile the Einstein-Podolsky-Rosen paradox [6]. We should, however, point out that our model's particles remain "classical", irrespectively of our knowledge of "hidden" parameters and the values which they take. On the other hand, sets of particles within the framework of the toy model can be subject to a statistical interpretation. Namely, two objects can exist in two different states even if they have the same values of "standard" three-dimensional parameters. However, they can still vary in values of "hidden" parameters, such as (for instance) the energy $U$. Perhaps, in the case of a multi-particle system one can speak of a probability distribution, characterizing those "hidden" parameters. However, rules controlling such distributions as well as possible reciprocal (mutual) variations of "hidden" parameters at the moment of interaction of objects which are characterized by those parameters, remain so far unknown.

It then seems that in the toy model we may interpret the probability rules of quantum mechanics as statistical results of a behaviour (or changes) of completely determined values of variables which are hidden to us. One may illustrate that on a simple example: the probability of finding a (nonrelativistic) "quantum" particle within some region of the configuration space is connected to the function $|D|^{2}$ integrated over the volume of this region. In general, the quantity $D$ denotes here the (normalized) wave-function representing the particle. However, in the case of a particle encountering potential(s) which are all time-independent, it is sufficient to assume that the quantity $D$ is the (normalized) spatial part of the wave-function representing this particle; see expression (17) which represents the simplest case of a wave-function - that for a free particle, i.e. a wave-function in the absence of any potentials. The toy model adds some new elements to the above picture coming from the "standard" (non-relativistic) quantum mechanics. Namely, the particle's wave-function occurring in quantum mechanics corresponds in the toy model to the function describing the particle's motion, i.e. also its oscillations in the additional dimension of spacetime. In turn, the function characterizing the motion of the toy-model particle depends, among others, on the value of the "hidden" parameter $U$ which is the particle's total energy in the five-dimensional spacetime; see the factors preceding the sine function in the formulae (13) and (14) for the simplest case of motion - that of a free particle. It is clear that two different objects which have identical values of all their directly observable parameters (such as the oscillation frequency $\omega$ ), may possess different values of the hidden, but inherent parameter $U$. Furthermore, one may also imagine a set of such apparently 
"identical" (non-interacting) particles which may have different (but always "completely" determined) values of the quantity $U$. In the case of a large set of such particles in the configuration space, it seems that the values of their "hidden" energies $U$ may be characterized by some probability distribution ${ }^{9}$ this probability distribution should then determine the value of the "hidden" energy $U$ of every single particle from the above-mentioned set of particles. Thus, the distribution of probability of finding a particle within some region of the configuration space, connected to the quantity $|D|^{2}$ in (non-relativistic) quantum mechanics, seems simply to result from, or to be determined by the probability distribution of the inherent parameter $U$.

Now, for the moment, let us consider a wave motion in general. From the Fourier analysis one knows that if the "dispersional" extent of the wave group in the $i$-th dimension equals $\Delta x_{i}$ and the indeterminacy, or "bandwidth" of the wave-vector in this particular dimension equals $\Delta k_{i}$, then both these quantities fulfil the following inequality

$$
\Delta x_{i} \Delta k_{i} \geq \frac{1}{2}
$$

it occurs for $i=2,3,4$. Likewise, if the impulse duration comprises the time interval $\Delta t$ and the frequency of the group is "spread over" the range $\Delta \omega$, then both these quantities fulfil the relationship

$$
\Delta t \Delta \omega \geq \frac{1}{2}
$$

Let us now return to the toy model and assume that all objects, whose behaviour we investigate here, move along their geodesic lines. We limit our considerations to the case of a given wave, or of a particle whose trajectory oscillates in the extra spatial dimension as well as to the case of a set of ordinary particles, i.e. those with negligible gravitational "interactions" among them. For the trajectory of the investigated object, we may write $\Delta \mathbf{p}=\hbar \Delta \mathbf{k}$ and $\Delta E=\hbar \Delta \omega$ where the quantities $\Delta \mathbf{p}$ and $\Delta E$ are defined analogically as

\footnotetext{
${ }^{9}$ It seems that in the considered case the probability distribution of the parameter $U$ could be estimated in a usual manner; for instance, one might imagine constructing a histogram: $n_{1}$ particles from the set take the values of the parameter $U$ from the interval $\left[U_{1}, U_{1}+\Delta U\right], n_{2}$ particles - from the interval $\left[U_{2}, U_{2}+\Delta U\right], \ldots$, and finally $n_{N}$ particles - from the interval $\left[U_{N}, U_{N}+\Delta U\right]$. Then, the formal limits $\Delta U \rightarrow 0$ and $N \rightarrow \infty$ remain to be performed and the probability $p=p(U) \equiv n / N(U)$ to be calculated.
} 
the quantities $\Delta \mathbf{k}$ and $\Delta \omega$ above, with taking into account the relationships $\mathbf{p}=\hbar \mathbf{k}$ and $E=\hbar \omega$ which hold for each of the monochromatic components of the particle's motion trajectory (of course, some of those monochromatic components may be "shifted" with respect to the spacetime $a=0$ and centred at some $a \neq 0$ ). Hence, according to expressions (201) and (21), we have

$$
\Delta x_{i} \Delta p_{i} \geq \frac{\hbar}{2} \quad \text { for } \quad i=2,3,4
$$

and

$$
\Delta t \Delta E \geq \frac{\hbar}{2}
$$

We can then assume that for a time interval $\Delta t$ sufficiently long, one has $\Delta E \cong 0$, or $E \cong$ const for our particles. ${ }^{10}$ Note that, from the standpoint of the considered model, there is nothing strange about the relationships (22) and (23). On the contrary, they are consistent with the dynamics of the particle's motion. For instance, as we were able to see earlier while analysing expression (14), the particle with the energy $E=0$ would move according to the formula $a= \pm U \eta$, instead of remaining motionless in the spacetime $a=0$. In effect, such a particle would irrevocably vanish from the spacetime $a=0$, without violating the conditions (22) and (23) which hold for the particle oscillating in the additional spatial dimension, i.e. for the one that periodically appears in the spacetime $a=0$. Moreover, one should note that, in our case, expressions (22) and (23) refer to the single particle travelling in the case of the metric (6) - with the harmonic motion, or moving in the presence of some potentials, as well as to a multi-particle system.

Let us now imagine performing such an experiment: Two plates are inserted in a very-low-temperature gas so that the noise and thermal motions of gas particles are minimized. We might imagine that the plates remain almost at rest in the spacetime $a=0 .\left({ }^{11}\right)$ Moving gas particles oscillate in the spatial dimension $a$ according to the formula (14), so between the two plates, only the particles which cross the hypersurface $a=0$ at the places $(a \approx 0, x, y, z)$ where the plates are situated, can rebound from the plates

\footnotetext{
${ }^{10}$ In other words, in order to make sure that the energy $E$ of the oscillating particle is the quantity remaining almost constant in time, one should observe the particle's motion for a sufficiently long (or "almost infinitely"long) interval of time $\Delta t$.

${ }^{11}$ For a macroscopic object one has $a \approx 0$, as the object consists of a large number of particles, between which the gravitational "interactions" are negligible, so the superposition of all the particles' oscillations is close to zero.
} 
and exist between them. Obviously, these would be the particles for which the quantity - half of a period of oscillations times the velocity with respect to the spacetime $a=0$ - fits the distance between the plates in natural numbers. Other particles simply circumvent the plate(s) in the space $a \neq 0$; see Fig. 1. Outside the plates all modes of the particles' oscillations are possible. This means that fewer particles will be found between than outside of the plates, so a net pressure will drive the plates together; and this seems to be nothing else but the Casimir effect.

In similar ways, one can analyse - with respect to our particles - diffraction, scattering, tunnelling into the classically forbidden region and other phenomena. The conclusion is quite clear - the toy particle, i.e. a purely classical relativistic particle moving along the geodesic line in the fivedimensional spacetime of the toy model, behaves in a way very similar to the quantum particle. However, in contrast to the real quantum particle, the toy particle's behaviour is very easy to explain, even with simple pictures; see, for instance, Fig. 1. This is because, in our case, the particle's behaviour is merely dictated by the geometry of the spacetime, in which the particle is moving. The conclusion of this section can then be expressed as follows: in our toy model, the quantum effects actually result from a purely classical structure of the "microspace". Quantum-mechanical effects, present in the toy model, are then the measurable consequence of the existence of the additional spatial dimension which is very hard to be detected directly. In other words, it seems that the entirely classical theory, which describes some phenomena occurring in the five-dimensional spacetime of the toy model, results in the theory of the quantum mechanics when one confines oneself to considering those phenomena as if they took place exclusively in the fourdimensional spacetime given by $a=0$; in this section we have seen that such an approach leads, among others, to the description of some purely corpuscular phenomena with the use of the wave-formalism.

Thus, equation (11) describes in the toy model a possible widest class of phenomena, ranging from the microworld to the entire Universe. The remaining question is whether we could allow ourselves to abandon the equations of quantum mechanics in the discussed model. It seems that we could not. Indeed, the problem of the quantum behaviour of a particle in a given situation might be settled by finding a solution to Eq. (11) with an appropriate stressenergy tensor. However, it is easy to imagine how difficult and arduous a task this would be. Therefore, a much more practical approach is to retain the Schrödinger (or rather the Klein-Gordon) or the Dirac equations, bearing 
in mind that a comprehensive and fully relativistic handling of a "quantum" issue would be provided by solving Eq. (II) with an appropriate stress-energy tensor. Thus, the Klein-Gordon or the Dirac equations can be, in the toy model, regarded as a kind of approach to the Einstein generalized equation (11) with a stress-energy tensor suitable for the investigated problem.

At the end of this section, let us note that ordinary particles (with finite masses) as well as massless ones should experience similar effects and phenomena. It is a consequence of the wave character of motion of both the particle types, which motion is described in both cases by very similar mathematical formalism; see the formulae (13) and (14). And indeed, there exist optical phenomena which are the exact analogues of each of the quantum effects related to the motion of a free particle with a finite mass.

Among numerous questions that arise as a result of the analysis of the particle's motion in the spacetime given by the metric ([6), a few come to the fore:

i) By what laws is the quantity $U$ governed? Does this quantity have any probability distribution in the multi-particle system? If so, do statistical distributions known from quantum mechanics reflect the distribution of the quantity $U$ ?

ii) Since, in our model, the quantum physics and its laws were not introduced directly, then what actually should be understood under the term "particle" in the toy model? In this situation, the only reasonable answer seems to be the model of a particle as a specific entity "formed from" the classical fields.

iii) What is the shape of "average" potentials with respect to the extra spatial dimension $a$ ? Is this shape similar for different potentials or is it dependent upon the potential type? Perhaps the shape of a potential with respect to the dimension $a$ is influenced by the spacetime structure in such a way that the shape is formed (or "forms itself") as a certain state of the dynamic equilibrium between the potential and the spacetime?

iv) How could charged particles be introduced into the discussed toy model or, in general, how could the electromagnetic interactions, as well as other interactions - weak or strong, be taken into account within the framework of this model? ${ }^{12}$ Does their presence result from specific spacetime

\footnotetext{
${ }^{12}$ Maybe, the first - and rather phenomenological - step in solving this task could consist in introducing into the toy model the five-dimensional wave equation and in investigating its properties; see Refs. [7, 8].
} 
deformations? Perhaps the answer to the last question is negative and therefore the electromagnetic, strong and weak interactions are transferred by the boson force carriers, instead of spacetime deformations.

In general, one may imagine that there exist two different types of solutions to the formally identical equation (11). The first type of solutions, represented by the metric tensor $g_{\mu \nu}$, would describe usual gravitational "interactions" which result from the spacetime deformations caused by the spacetime distribution of matter/energy. This type of solutions is actually investigated in the present paper and composes the contents of the toy model. The second type of solutions to equation (II) would correspond to all kinds of "typical" (i.e. not gravitational) interactions: the strong, weak, and electromagnetic ones (and, maybe, others - not detected so far). These Kaluza-Klein-type solutions would be in the form of a "potential" tensor (i.e. in the form of a tensor of "potentials") with components characterizing the above-mentioned interactions, such as the four-vector potential $A_{\mu}$ for the electromagnetic forces. The second type of solutions might possibly be subject to some quantization-like procedure(s). Maybe, there also exists the third type of solutions to the Einstein equation (11), which would describe the (internal) structure of elementary particles.

\section{Schwarzschild metric}

The aim of this section is to check whether the toy model introduces any corrections to the predictions of the "standard" general relativity regarding, among others, the so-called "five tests of general relativity"; see Ref. 9]. All these tests are a consequence of a particular solution to the Einstein original equation, known as the Schwarzschild metric. Let us consider how this metric will be affected by the introduction of the extra spatial dimension.

In the presence of a spherically symmetric object with a mass $M$ in an empty (asymptotically flat) space, an exact solution of equation (11) with the stress-energy tensor given by expression (2) takes the following form,

$$
\begin{aligned}
d s^{2}=(1 & \left.-\frac{2 m_{M}}{r}\right)\left(1+|\lambda| a^{2}\right) c^{2} d t^{2}-\left(1+|\lambda| a^{2}\right)^{-1} d a^{2} \\
& -\left(1-\frac{2 m_{M}}{r}\right)^{-1} d r^{2}-r^{2} d \theta^{2}-r^{2} \sin ^{2} \theta d \varphi^{2}
\end{aligned}
$$

where $r \in(0, \infty), \theta \in[0, \pi]$, and $\varphi \in[0,2 \pi)$ are the polar coordinates of the "macroscopic" three-dimensional space around the mass $M$, and $m_{M} \equiv$ 
$G M / c^{2}$. In the field of a spherically symmetric object with the mass $M$, a solution to the equation of motion of a test particle with respect to the dimension $a$ can be written as

$$
a(\eta)= \pm \sqrt{\frac{U^{2}-E^{2}}{E^{2}|\lambda|}} \sin (\omega \eta)
$$

where

$$
\omega \equiv \frac{E}{\hbar}\left(1-\frac{2 m_{M}}{r}\right)^{-1 / 2},
$$

the quantity $\eta$ is an affine parameter, and $E$ denotes the particle's "threedimensional" energy, defined exactly in the same form as in the case of the Schwarzschild original solution; then - for instance - for a particle with a finite (non-zero) mass $m$ one has

$$
\begin{array}{r}
E^{2}=c^{2}\left(1-\frac{2 m_{M}}{r}\right)\left[m^{2} c^{2}\right. \\
\left.+\left(1-\frac{2 m_{M}}{r}\right)^{-1}\left(p^{r}\right)^{2}+r^{2}\left(p^{\theta}\right)^{2}+r^{2}\left(\sin ^{2} \theta\right)\left(p^{\varphi}\right)^{2}\right]
\end{array}
$$

where $p^{r} \equiv m d r / d \tau, p^{\theta} \equiv m d \theta / d \tau, p^{\varphi} \equiv m d \varphi / d \tau$, and $\tau$ denotes the proper time of the particle whose motion is under consideration. Recall from the previous section that, for sufficiently large time intervals, the quantity $E$ remains almost constant for a particle moving along the geodesic line.

Below, it is assumed that the mass $M$ of the investigated object or system of objects is small when compared with its size: $2 G M /\left(c^{2} r\right) \ll 1$.

\subsection{Five tests of general relativity}

Gravitational redshift. The general theory of relativity predicts that the spectrum of the radiation emitted in the neighbourhood of a massive object and receding from it, should be shifted towards the red part of the spectrum. In turn, the spectrum of light moving in the direction of such an object should show shift towards the violet part of the spectrum.

Let us suppose that an electromagnetic wave or a wave of matter has a frequency $\omega_{1}$ in the distance $r_{1}$ from the mass $M$. In the distance $r_{2}$ from this object the wave frequency is $\omega_{2}$. Then, according to the formula (26), the gravitational redshift defined as $z \equiv\left(\omega_{1}-\omega_{2}\right) / \omega_{2}$ is given by

$$
z=\left(\frac{1-2 m_{M} / r_{2}}{1-2 m_{M} / r_{1}}\right)^{1 / 2}-1 \cong m_{M} \frac{r_{2}-r_{1}}{r_{1} r_{2}} \approx \frac{g h}{c^{2}},
$$


where $g \equiv G M / r^{2}$ (with some $r$, outside the mass $M$, such that $r_{1} \approx r \approx r_{2}$ ) and $h \equiv r_{2}-r_{1}$. The above formula is identical to that obtained on the basis of the considerations concerning the energy conservation for a particle (or wave) moving along the geodesic line in the four-dimensional $(1+3)$ spacetime of the "standard" general relativity, described by the Schwarzschild original metric. It should be stressed, however, that in the standard theory the explicit relationship - such as the formula (26) in the toy model - between the frequency $\omega$ of the particle's oscillations and the "field" factor $\left(1-2 m_{M} / r\right)$ does not exist. It happens, since there is no direct connection between the quantity $\omega$ and the spacetime curvature in the "standard" general relativity.

The remaining well-known tests of general relativity concern propagation delay of radar signals, light deflection, perihelion precession and geodesic precession. After some calculations one can state that the values of all measurable parameters, characteristic for the above-mentioned processes or phenomena, derived within the framework of the toy model are the same as those obtained for the case of the Schwarzschild original metric within the "standard" general relativity.

\subsection{Dark matter}

In this section, we argue that there are no reasons for postulating the existence of dark objects. We also try to show that effects which are usually attributed to the presence of a hypothetical non-luminous matter can be explained readily by geometric properties of a spacetime which contains an additional spatial dimension denoted by $a .\left({ }^{13}\right)$

Presumably the most "spectacular" need for a dark material is provided by the observational data concerning spiral galaxies. These objects seem to have a constant circular rotation velocity $v_{c}$, independently of the galaxy radius $R$ (apart from the relatively small, initial values of $R$ when, obviously, $v_{c}$ approaches zero as $R \rightarrow 0$ ). On the other hand, the brightness of matter - and the density of a luminous material, if we reasonably assume that the brightness-to-density ratio remains constant - in spiral galaxies decreases exponentially with growing radius $R$. Simple calculations based on the Newtonian mechanics suggest, however, that for a constant circular velocity $v_{c}$ the density of matter should decrease as $1 / R^{2}$, i.e. much more slowly than

\footnotetext{
${ }^{13}$ It is worth adding that some authors have suggested solutions to the missing material problem, by the manifest changing of Einstein's law of gravity [10, 11, 12.
} 
exponentially [1]. One therefore assumes that, in addition to the "ordinary" visible matter, there also exists a dark material that causes the observational data to remain in agreement with reasonable (for example, on a scale of the solar system) predictions of the Newtonian mechanics. The dark matter should be concentrated in outer parts of a galaxy, forming the so-called dark halo, as the Newtonian calculations and astronomical observations give practically identical results only for small (initial) values of the galaxy radius $R$. Such a situation seems to be a general feature of all the known spiral galaxies. This is, given the dark matter, somewhat weird, since both the luminous and the dark material should possess identical gravitational properties. Thus, both kinds of the material should display - independently of one another rather random spatial distribution in different observed galaxies. Therefore, for various galaxies a completely different dark matter distribution inside the galactic space might be expected.

There are also other reasons to postulate the existence of dark matter [1, 10]. In this paper, however, we will focus on probably the most spectacular case, i.e. that of spiral galaxies. Other cases such as those concerning the motion of so-called dwarf galaxies [1] as well as of regular clusters of galaxies [13, 14] which are in a virial equilibrium, may be approached in a very similar manner.

For the purpose of studying large astronomical systems, such as spiral galaxies, usually the so-called virial theorem is being applied [10]. In the case of the Newtonian mechanics, the virial theorem gives

$$
\frac{G M}{R}=2 \sigma^{2}
$$

where $M=M(R)$ is the mass contained within the gravitational radius $R$ and $\sigma$ denotes the velocity dispersion; see, for instance, Refs. 1, 10. Note that for a spiral galaxy one has $v_{c}=2^{1 / 2} \sigma$. As it was pointed out earlier, equation (29) implies - if $v_{c}(R)=$ const is assumed - the spatial distribution of the mass of a spiral galaxy which is inconsistent with observations. Now let us note that the virial theorem applied in the toy model assumes a form slightly different from Eq. (29), namely

$$
\frac{G M}{R}+\frac{c^{2}}{2}|\lambda| a^{2}=2 \sigma^{2}
$$

for $|\lambda| a^{2} \ll 1$. Due to the rapid oscillations of the quantity $|\lambda| a^{2}$ in time, one will detect an "expected", i.e. mean (or average) value of this quantity 
over time. Thus, in the formula (30), the quantity $|\lambda| a^{2}$ should rather be substituted by its average value,

$$
\left\langle|\lambda| a^{2}\right\rangle_{\tau}=\frac{U^{2}-E^{2}}{2 E^{2}} \equiv U_{E}
$$

Note that for a value of the energy $E$ close to $U$, we have $U_{E} \approx(U-E) / E$.

Asking whether the value of the quantity $U_{E}$ can depart considerably from zero for a large macroscopic object, such as a planet or even a part of a galaxy, should start the analysis of Eq. (30). In order to answer this question, let us return for a moment to the formulae (14) or (25). Note that the "classical" motion of a particle in the four-dimensional spacetime $a=0$ of the toy model corresponds in the discussed case to the performing of a formal limit $|\lambda| \rightarrow \infty$. Note also that this operation is equivalent to taking of the limit $\hbar \rightarrow 0$, as one has $|\lambda| \propto \hbar^{-1}$. Thus, within the "classical" limit we obtain $|\lambda| \rightarrow \infty$, and consequently $a(\eta) \rightarrow 0$. However, the product $|\lambda| a^{2}$ can remain a finite, non-zero number.

Let us recall expression (31); we see that in the case of studying the virial theorem in the context of dark matter, there again arises the problem of the meaning of the total energy $U$. The first question that emerges is whether the quantity $U_{E}$ depends on parameters - such as the mass - of the investigated object and, if so, how it depends on it. It seems possible that $U_{E}$ could take relatively large values for "quantum" particles, i.e. for particles which have relatively low masses and/or energies $E$ [see the formula (19)], and very small values for "classical" massive objects (see footnote 11 in section 3.1), although this does not have to always be true and remain a rule.

Now let us make a digression referring to the mass amount. In general, the maximum acceptable mass of a single particle seems to be the Planck mass $m_{P l} \equiv h /\left(c^{2} T_{l}\right)$; see section 3 and the formula (8) therein. Note that an object with the Planck energy $E_{P l} \equiv m_{P l} c^{2}$, in the anti-de Sitter spacetime $\mathcal{S}^{1} \times \mathcal{R}^{1}$ whose covering surface is given by the metric (17), oscillates in this spacetime with a vibration period equal to the Planck time $T_{l}=h / E_{P l}$. A very interesting relationship can be observed: namely, objects from this anti-de Sitter spacetime, which have vibration periods equal to $n T_{l}$, where $n \in \mathcal{N}$, retrace their own life histories exactly each time after the lapse of the coordinate time $n T_{l}$. This relationship - which is a condition of the particles' time-like (geodesic) curves closure in the anti-de Sitter spacetime $\mathcal{S}^{1} \times \mathcal{R}^{1}$ - could be called the resonance condition of the particles' vibration 
in the anti-de Sitter spacetime. Note that in the discussed phenomenon, the Bohr-Sommerfeld quantum condition(s) are satisfied, as we have

$$
\oint p_{q} d q=\oint \frac{h}{\Lambda} d(c t)=\frac{h}{c n_{q} T_{l}} c n_{q} T_{l}=h
$$

where the quantities $\Lambda$ and $c$ denote, respectively, the wavelength and the velocity of the oscillating particle with respect to the hypersurface $a=0$, i.e. in the $(c t)$-direction of the anti-de Sitter spacetime.

Let us now turn to our original problem of dark matter and assume that the distribution of the mass density is consistent with observational data, i.e. it exponentially decreases with growing galaxy radius $R$. For sufficiently large distances $R$ from the spiral galaxy centre, a component $G M / R$ on the left-hand side of Eq. (30) takes a (relatively) small value, so we assume that it becomes rather negligible as compared with $c^{2}\left\langle|\lambda| a^{2}\right\rangle_{\tau} / 2$. On the other hand, for distances $R$ sufficiently large, the relative growth in the mass of the galaxy $\Delta M / M$ within the radius $R$, along with increasing $R$ by $\Delta R$, is relatively small. In such a case, also the value of the relative increase in the momentum $\Delta p / p$ - along with the growth of $R$ by $\Delta R$ - is comparatively small, as the circular velocity of the galaxy is close to constant for large values of $R$. From this it immediately follows that the corresponding relative increments in the energies $\Delta E / E$ and - as a consequence of all that was said above - also $\Delta U / U$ are comparatively small, too; ${ }^{14}$ see expressions (25) and (27), where for the considered case one has $p^{r}=0=p^{\theta}$ and the circular velocity $v_{c}$ is given by the relationship $v_{c}=r(\sin \theta) p^{\varphi} / m$. Therefore we can assume - on the basis of the relation (31) - that for relatively large distances $R$, we have $\left\langle|\lambda| a^{2}\right\rangle_{\tau} \approx$ const. Thus, there holds

$$
\frac{c^{2}}{2}\left\langle|\lambda| a^{2}\right\rangle_{\tau} \approx v_{c}^{2}(R) \approx \text { const } .
$$

For a typical spiral galaxy, the constant circular velocity is of the order of $10^{2} \mathrm{~km} \mathrm{~s}^{-1}$. Then

$$
\left\langle|\lambda| a^{2}\right\rangle_{\tau}=U_{E} \sim 10^{-7}
$$

which seems to be a reasonable result considering that the discussed system seems to be highly "classical".

\footnotetext{
${ }^{14}$ It seems to be rather improbable, also from the statistical point of view, that the "hidden" energy $\Delta U$ of the mass $\Delta M$ of the outer part of the galaxy - for which $\Delta M / M \approx$ 0 and $\Delta E / E \approx 0-$ does not fulfil the condition $\Delta U \ll U$.
} 
Now we will examine what happens within the small distance from the galaxy centre. Assuming that $R \rightarrow 0$ we obtain $|\lambda| a^{2} \rightarrow 0$, as for $R \rightarrow 0$ the relation $U \rightarrow E \rightarrow 0$ takes place; see the formula (25). It is because for $R=0$ obviously holds $U=E=0$ and $a(\eta)=0$ (as there is no mass right in the middle point of a spiral galaxy, since the topological measure of a point in a three-dimensional space or of a one-dimensional line in a four-dimensional space is equal to zero), whereas we do not expect the function $a(R)$ to be not continuous. It seems that the quantity $\left\langle|\lambda| a^{2}\right\rangle_{\tau}$ is a monotonic function of $R$, reaching plateau for sufficiently large values of $R$, for which - along with the further growth of $R$ - the value of the quotient $U / E$ remains virtually on the same level, at least as compared with the rate of the earlier growth.

Let us note that due to the presence of the "hidden" parameter $U$ of an unknown distribution $U=U(M, R)$ in the quantity $\left\langle|\lambda| a^{2}\right\rangle_{\tau}$, theoretical results can in principle be perfectly fitted to experimental data. In other words, the comparison between observational results and Eq. (30) might allow us to determine the function $U=U(M, R)$. One of many possibilities of a very precise representation of experimental data by Eq. (30) is provided by the following relationship

$$
\left\langle|\lambda| a^{2}\right\rangle_{\tau}=\alpha R
$$

where $\alpha$ seems to be a "universal" constant, the value of which, $\alpha \sim 10^{-28}$ $\mathrm{m}^{-1}$, allows one to reproduce to some extent the experimental data for numerous large astronomical systems of various types and sizes. ${ }^{15}$ Let us note that the potential given by the formula (35) evidently does not satisfy the relationship (33), as for expression (35) one has $U^{2}=E^{2}(1+2 \alpha R)$. The existence of such a relationship, however, seems to be far less possible than of expression (33) which implies that $U / E \approx$ const for sufficiently large values of the quantity $R$.

In a similar manner to that described above we can explain phenomena attributed to the presence of dark matter in astronomical objects other than spiral galaxies, e.g. in so-called regular clusters of galaxies as well as in dwarf

\footnotetext{
${ }^{15}$ Detailed investigations of a number of spiral galaxies, with taking into account a linear contribution to the potential function, were performed by Mannheim [15] 16]; note that in Ref. [16] one assumes that $\alpha \equiv \alpha_{1}+N \alpha_{2}$ where $\alpha_{1}$ and $\alpha_{2}$ are universal constants and $N$ denotes the total amount of visible - stellar (and gaseous) - matter in solar mass units in a galaxy. The presence of the potential given by the right-hand side of expression (35) in the virial equation (30) also permits one to reproduce the experimental data concerning both regular clusters of galaxies and dwarf spheroidal galaxies.
} 
spheroidal galaxies [1. Hence, the presence of the extra spatial dimension enables the explanation of the dynamics of large astronomical objects without introducing a substantial amount of any dark matter. Of course, it does not rule out possibility that a part of the matter in the investigated objects exists in a non-luminous form, e.g. as a cold dark matter and/or a hot one. However, the volume of the "missing mass" necessary to explain - together with the luminous matter - the dynamics of large astronomical objects, considerably exceeds the masses of so far discovered potential components of cold as well as hot dark matter. Nevertheless, it seems that the toy model is able to supplement these deficiencies, solving the problem of the missing mass without introducing any additional dark matter in excess of that whose existence has already been proved by observation.

\section{Cosmology}

\section{$5.1 \quad$ Field equations}

In this section we will examine, how the currently accepted cosmological models are influenced by the introduction of the extra spatial dimension. To focus our attention we will consider exclusively the Friedmann-RobertsonWalker model(s) 9]. We suppose that our model, i.e. the cosmological toy model, will satisfy a cosmological principle which assumes that the Universe is spatially homogeneous and isotropic, but only for the three "macroscopic" spatial dimensions. Such a toy universe is described by the metric

$$
\begin{array}{r}
d s^{2}=\left(1+|\lambda| a^{2}\right) c^{2} d t^{2}-\left(1+|\lambda| a^{2}\right)^{-1} d a^{2} \\
-[R(t)]^{2}\left[\left(1-k r^{2}\right)^{-1} d r^{2}+r^{2} d \theta^{2}+r^{2} \sin ^{2} \theta d \varphi^{2}\right],
\end{array}
$$

where $r \in[0, \infty), \theta \in[0, \pi]$, and $\varphi \in[0,2 \pi)$ are the dimensionless polar "comoving" coordinates, the curvature parameter $k$ is a constant equal to 0 or to \pm 1 , and $R(t)$ denotes the cosmic scale factor, or the expansion parameter.

In order to solve equation (11) with the above metric and thus to determine conditions which should be satisfied by the scale factor $R(t)$, the stressenergy tensor $\widetilde{T}_{\mu \nu}$ for the investigated problem must be appropriately defined. We assume that

$$
\widetilde{T}_{\mu \nu}=T_{\mu \nu}+\widehat{T}_{\mu \nu},
$$


where

$$
\begin{array}{ll}
\widehat{T}_{\mu \nu}=\left(\varrho+\frac{\tilde{p}}{c^{2}}\right) u_{\mu} u_{\nu}-\widetilde{p} g_{\mu \nu} \quad \text { for } \mu=0,1 \quad \& \quad \nu=0,1 \\
\widehat{T}_{\mu \nu}=\left(\varrho+\frac{p}{c^{2}}\right) u_{\mu} u_{\nu}-p g_{\mu \nu} \quad \text { otherwise },
\end{array}
$$

and $T_{\mu \nu}$ is defined by expression (2), while $\varrho$ denotes the rest-frame density of matter/energy of the fluid filling the Universe, $p$ is the fluid (rest-frame) isotropic pressure along the three "macroscopic" spatial dimensions, and $\widetilde{p}$ denotes the pressure of matter (or energy) along the extra spatial dimension $a$, i.e. the pressure acting orthogonally (on)to hypersurfaces of constant values of the coordinate $a$. In turn, $u^{\mu}=d x^{\mu} / d \tau$ is the fluid proper "five-velocity" (along, or with respect to, the $\mu$-th coordinate; $\tau$ denotes the proper time), where $x^{\mu}(\tau)$ is the world-line of a fluid element. Note that, in general, the pressures $p$ and $\widetilde{p}$ should not be equal to one another as we assume spatial homogeneity and isotropy of the Universe for the three "macroscopic" spatial dimensions only.

Inserting the metric (36) into the Einstein equation (11) with the stressenergy tensor given by expression (37), one obtains the field equations which must be satisfied by the cosmic scale factor $R(t)$,

$$
\begin{aligned}
c^{2} k A+\dot{R}^{2} & =-\frac{\kappa}{3} c^{2} \widehat{T}_{00} R^{2} \\
c^{2} k A+\ddot{R} R+\dot{R}^{2} & =\frac{\kappa}{3} c^{2} \widehat{T}_{11} A^{2} R^{2} \\
r^{2}\left(c^{2} k A+2 \ddot{R} R+\dot{R}^{2}\right) & =\kappa c^{2} \widehat{T}_{33} A,
\end{aligned}
$$

where we have introduced the following notation: $R \equiv R(t), \dot{R} \equiv d R(t) / d t$, $\ddot{R} \equiv d^{2} R(t) / d t^{2}$, and $A \equiv 1+|\lambda| a^{2}$. Obviously, the three spatial equations for the "macro-dimensions" $x^{2} \equiv r, x^{3} \equiv \theta$ and $x^{4} \equiv \varphi$ are equivalent to each other, so only one of them - namely (42) - is written explicitely here. In an (interesting to us) temporarily co-moving coordinate system, for which $a=$ const and $x^{i}=$ const, where $i=2,3,4$, we obtain

$$
\begin{aligned}
c^{2} k A+\dot{R}^{2} & =-\frac{\kappa}{3} c^{4} \varrho A R^{2} \\
c^{2} k A+\ddot{R} R+\dot{R}^{2} & =\frac{\kappa}{3} c^{2} \widetilde{p} A R^{2} \\
c^{2} k A+2 \ddot{R} R+\dot{R}^{2} & =\kappa c^{2} p A R^{2} .
\end{aligned}
$$


Since we assume that the quantity $R$ (i.e. the scale factor) is exclusively a function of the coordinate time $t$ and not of the dimension $a$, we must assume that $k=0$ over the whole history of the Universe, i.e. for times $t \geq 0$. This is because an assumption that $k= \pm 1$ inevitably results in a dependence of the factor $R$ on the dimension $a$, or one has $\varrho(a \rightarrow \pm \infty) \neq 0$, which we would like to avoid. Moreover, if the scale factor $R$ is to be a function of time $t$ only and the condition $k=0$ is assumed, then also the quantities $\varrho A, \widetilde{p} A$ and $p A$, present in Eqs. (43)-(45), should not depend on $A$. Thus, expressions determining the matter (or energy) density $\varrho$ as well as the pressures $\widetilde{p}$ and $p$ of the fluid filling the Universe in a function of the extra spatial dimension $a$ should take the following form,

$$
\begin{aligned}
& \varrho(t=\text { const }, a) \sim A^{-1} \\
& \widetilde{p}(t=\text { const }, a) \sim A^{-1} \\
& p(t=\text { const }, a) \sim A^{-1} .
\end{aligned}
$$

It is clear that the above relationships reflect geometric properties of the toymodel spacetime which is hyperbolically curved along the additional spatial dimension $a .\left({ }^{16}\right)$ In effect, we have

$$
\begin{aligned}
& \varrho(t, a=0)=A \varrho(t, a) \\
& \widetilde{p}(t, a=0)=A \widetilde{p}(t, a) \\
& p(t, a=0)=A p(t, a) .
\end{aligned}
$$

It can be easily proved that if the system of equations (43)-(45) is to be self-consistent for any values of $R$ and $A$, then the following condition must be satisfied,

$$
3 p-2 \widetilde{p}=c^{2} \varrho .
$$

Let us suppose that the fluid filling the present Universe is basically composed of matter and that this matter exists in the form of dust, which means that its pressure $p$ is negligible as compared with the value of $c^{2} \varrho$, i.e. one has $c^{2} \varrho \gg p \approx 0$. As a result we obtain the relationship $\varrho=-2 c^{-2} \widetilde{p}$. Obviously, this relationship - meaning negative value of the pressure $\widetilde{p}-$ is an effect of the spacetime geometry of the proposed toy model rather than of

\footnotetext{
${ }^{16}$ Note that the particles of an ideal fluid which possess a very small energy $E$ can penetrate relatively deep into the dimension $a$ [see the formula (19)], so expressions (46)(48) do not seem to be unrealistic.
} 
properties of the matter alone; the dependence of the quantities $\varrho, p$ and $\widetilde{p}$ on the geometry of the spacetime is especially explicitely seen in expressions (46)-(48). Furthermore, let us note that for radiation satisfying relationship $p=c^{2} \varrho / 3$ one has $\widetilde{p}=0$.

It is also easy to show that in the weak-field approximation, under the condition that $c^{2} \varrho \gg p \approx 0$ and for $A \approx 1$, the $(0,0)$-component of the Einstein equation (11) with the stress-energy tensor defined by expression (37) approaches - while taking into account the condition (52) - the Poisson equation, $\nabla_{\mathbf{r}}^{2} \psi \approx 4 \pi G \varrho$; see also section 2 .

Now we consider the (local) energy conservation, or continuity equation for the fluid characterized by the stress-energy tensor $\widetilde{T}_{\mu \nu}$; this equation takes the same form as in the case of the standard Friedmann model,

$$
\frac{d \varrho}{d t}+\left(\varrho+\frac{p}{c^{2}}\right) \frac{3 \dot{R}}{R}=0 .
$$

We assume that the fluid which fills the present Universe is mainly composed of matter existing in the form of dust. Thus we obtain

$$
\frac{d \varrho}{d t}+\varrho \frac{3 \dot{R}}{R}=0
$$

which gives

$$
\varrho(t, a=\text { const }) R^{3}=\text { const } .
$$

For radiation, which fulfils the equation of state $p=c^{2} \varrho / 3$, we have

$$
\varrho(t, a=\text { const }) R^{4}=\text { const } .
$$

Let us define the Hubble constant $H(t) \equiv \dot{R}(t) / R(t)$, the critical density of matter

$$
\varrho_{c} \equiv-\frac{3 H^{2}}{\kappa c^{4} A},
$$

and the current critical density of matter

$$
\varrho_{c 0} \equiv \varrho_{c}\left(t_{0}\right)=-\frac{3 H_{0}^{2}}{\kappa c^{4} A}=\frac{3 H_{0}^{2}}{8 \pi G A}
$$

where $H_{0} \equiv H\left(t_{0}\right)$ denotes the present value of the Hubble constant. Let $\varrho_{0}$ represents the density of matter in the present Universe, so $\varrho_{0} \equiv \varrho\left(t_{0}\right)$. 
We can also define a parameter $\Omega$, frequently used in cosmology, as

$$
\Omega \equiv \frac{\varrho}{\varrho_{c}},
$$

so we have

$$
\Omega=-\frac{\kappa c^{4}}{3 H^{2}} \varrho A .
$$

Furthermore, the present value of the density parameter $\Omega$ reads

$$
\Omega_{0} \equiv \Omega\left(t_{0}\right)=\frac{\varrho_{0}}{\varrho_{c 0}} .
$$

It can be easily noticed that for a given density of matter/energy $\varrho$, the parameter $\Omega$ varies by a factor of $A$ with respect to its definition in the standard model, since we have

$$
\Omega^{\text {stand }}=-\frac{\kappa c^{4}}{3 H^{2}} \varrho,
$$

and then one obtains

$$
\Omega=A \Omega^{\text {stand }} .
$$

It is of course an effect of the modification of the critical density value with respect to its definition in the original Friedmann model,

$$
\varrho_{c}=A^{-1} \varrho_{c}^{\text {stand }} .
$$

Let us remind the reader that the assumption $R=R(t)$ - so $R$ is independent of the coordinates other than $t$ - leads to the conclusion that the value of $k$ is equal to 0 . Therefore - from Eq. (43) - one can obtain an important relationship,

$$
\Omega=1 .
$$

Hence, the density of matter/energy in the Universe assumes its critical value, meaning that the geometry of the three "macroscopic" spatial dimensions of the Universe is flat. Obviously, the flat geometry of these "macroscopic" spatial dimensions is a consequence of the assumption that $k=0$, which de facto is equivalent to the formula (65).

Note that according to the equalities (63) and (65)), the following can be written,

$$
\Omega^{\text {stand }}=A^{-1} .
$$


Then a question arises as to whether - bearing in mind relations (65) and (66) - we can estimate the value of the cosmological density parameter defined as in the standard model, i.e. $\Omega^{\text {stand }}$. It seems to be difficult as we got used to the problems with the estimation of the value of the factor $A$. At this place we should appeal to the experiment ${ }^{17}$ and observe that while experimentally estimating the value of the quantity $\Omega^{\text {stand }}$ applying the formula (62), the density of matter $\varrho$ is usually calculated as, roughly speaking, the quotient of the detected mass - i.e. of the mass which is subject to our perception - by the (three-dimensional) volume of the space with $a=0$, within which (i.e. for $a=0$ ) as well as around which (i.e. for $a \neq 0$ ) the detected mass is situated. The value of the factor $A$ in the formula (66) should then correspond to the range, or width of the additional spatial dimension $a$ (around $a=0$, on both sides of $a$, i.e. for $\pm a$ ) which is observable, or rather detectable indirectly, i.e. by detecting the mass which is situated there. Let us note that $\Omega \geq \Omega^{\text {stand }}$ always, since $A \geq 1$, as one has $|\lambda| a^{2} \geq 0$. We expect that for the present Universe as a whole, the quantity $A$ could be of the order of $10^{1}$, or even of $10^{2}$. Namely, for such values of $A$ the density of matter $\varrho(A=10)$ is, according to the formula (49), an order of magnitude lower than the density of matter for $A=1$, i.e. for $a=0$. Thus, the visibility of the matter situated at $A=10$ - or the perception of it - is much weaker than that of the matter concentrated at $A=1$. In other words, one has $\varrho\left(t, A_{d}=10\right)=10^{-1} \varrho(t, A=1)$, so most matter - with respect to the dimension $a$ - is situated in the region of space around $a=0$, within the interval $a \in\left[-a_{d}, a_{d}\right]$, where $A_{d} \equiv 1+|\lambda| a_{d}^{2}$. Thus, according to the formula (66) , it could be assumed that

$$
\Omega^{\text {stand }} \sim A_{d}^{-1}=10^{-1} .
$$

One may suppose that the given above value of $A_{d}$ - one order of magnitude greater than $A(a=0)$ - should roughly correspond to the range of values of the additional spatial coordinate, given by $a \in\left[-a_{d}, a_{d}\right]$, which is subject to our direct perception.

\footnotetext{
${ }^{17}$ Note that in the toy model the quantity $\Omega$ only is of an essential theoretical significance, whereas $\Omega^{\text {stand }}$ is the quantity of a rather purely experimental relevance. As one may see, there exist two approaches to estimating the "actual" value of the quantity $\Omega^{\text {stand }}$ : the first one - experimental - on the basis of the formula (62), and the second one - "theoretical" - with the use of expression (66). Both the approaches should in principle give similar results. In practice, however, they can differ depending e.g. on which is the method used to estimate the density of matter $\varrho$; see section 5.2 .
} 
Another, more direct and probably more appropriate approach to estimating the value of the quantity $\Omega^{\text {stand }}$ could proceed as follows: Let us assume that the quantities $\pm a_{d}$ correspond to the limits of the directly observable interval of the dimension $a$ around $a=0$, so one has $\Omega^{\text {stand }}=A_{d}^{-1}=$ $\left(1+|\lambda| a_{d}^{2}\right)^{-1}$. If we reasonably assume that $a_{d}=L=2 \pi /|\lambda|^{1 / 2}$, then we obtain

$$
\Omega^{\text {stand }}=\left(1+4 \pi^{2}\right)^{-1} \cong 0.025
$$

One more approach to estimating the value of the parameter $\Omega^{\text {stand }}$ - the most thorough and perhaps the most promising - could be imagined: Let us assume that all the observable matter is concentrated within the interval of the additional spatial dimension given by $a \in\left[-a_{d}, a_{d}\right]$ or, in other words, within the interval $A \in\left[1, A_{d}\right]$ "taken twice", as one has $A_{d}=1+|\lambda|\left( \pm a_{d}\right)^{2}$. We may then write

$$
\Omega^{\text {stand }} \propto 2 \int_{1}^{A_{d}} \varrho_{a} d A=2 \varrho_{a=0} \int_{1}^{A_{d}} A^{-1} d A=2 \varrho_{a=0} \ln A_{d}
$$

where $\varrho_{a} \equiv \varrho(a)$. In the case of the above expression, for the normalization factor one can take

$$
\Omega \propto 2 \int_{1}^{A_{d}} \varrho_{a} A d A=2 \varrho_{a=0} \int_{1}^{A_{d}} d A=2 \varrho_{a=0}\left(A_{d}-1\right)
$$

note that, while obtaining both the above formulae, we have applied the equality (49) which results, for instance, in the relationship $A d \varrho_{a}=-\varrho_{a} d A$ for $t=$ const. Dividing expressions (69) and (70) by one another and taking into account the formula (65), we find that

$$
\Omega^{\text {stand }}=\frac{\ln A_{d}}{A_{d}-1} .
$$

If we then assume that $a_{d}=L$, so $A_{d}=1+4 \pi^{2}$, we obtain

$$
\Omega^{\text {stand }}=\frac{\ln \left(1+4 \pi^{2}\right)}{4 \pi^{2}} \cong 0.094
$$

However, if we demand the value of $A_{d}$ to fulfil the condition $\varrho\left(t, A_{d}\right)=$ $10^{-1} \varrho(t, A=1)$, then we obtain that $A_{d}=10$, and from the formula (71) we have

$$
\Omega^{\text {stand }} \cong 0.256
$$


which remains in agreement with the commonly accepted value of the cosmological mass density parameter $\Omega_{0}^{\text {stand }}$ that incorporates a contribution usually attributed to the existence of dark matter; see, for instance, Ref. [17] and references therein. In the toy model, such a contribution is a consequence of the existence of the additional spatial dimension $a$, and this has been explicitely taken into account while calculating the above value of the cosmological density parameter $\Omega^{\text {stand }}$; see also sections 4.2 and 5.2. Thus, the above considerations indicate, to some extent, the self-consistency of the model proposed in this paper.

However, we can also calculate the cosmological density parameter $\Omega^{\text {stand }}$ in a quite similar manner as above, but with the appropriate integrations performed now over the quantity $d a$ instead of $d A$,

$$
\begin{aligned}
\Omega^{\text {stand }} & \propto \int_{-\sqrt{|\lambda|} a_{d}}^{+\sqrt{|\lambda|} a_{d}} \varrho_{a} d(\sqrt{|\lambda|} a)=2 \varrho_{a=0} \arctan \left(\sqrt{|\lambda|} a_{d}\right) \\
\Omega & \propto \int_{-\sqrt{|\lambda|} a_{d}}^{+\sqrt{|\lambda|} a_{d}} \varrho_{a} A d(\sqrt{|\lambda|} a)=2 \varrho_{a=0} \sqrt{|\lambda|} a_{d},
\end{aligned}
$$

so after the normalization of the parameter $\Omega$ to unity one arrives at the formula

$$
\Omega^{\text {stand }}=\frac{\arctan \left(\sqrt{|\lambda|} a_{d}\right)}{\sqrt{|\lambda|} a_{d}} .
$$

Assuming that $a_{d}=L$, so $|\lambda|^{1 / 2} a_{d}=2 \pi$, we obtain $\Omega^{\text {stand }} \cong 0.225$, whereas for $A_{d}=10$, that is when $|\lambda|^{1 / 2} a_{d}=3$, one has $\Omega^{\text {stand }} \cong 0.416$. It is then clear that the whole above discussion concerning the estimation of the actual value of the cosmological density paramater $\Omega^{\text {stand }}$ seems to confirm the fact that this quantity is not of essential relevance in the toy model.

Now let us define a quantity called the deceleration parameter,

$$
q \equiv-\frac{\ddot{R} R}{\dot{R}^{2}} .
$$

According to Eqs. (43) and (45), in the case of dust filling the Universe for which $p=0$ and $\widetilde{p}=-c^{2} \varrho / 2$, there holds the relationship $q=\Omega / 2$. In turn, one finds $q=\Omega$ for radiation. 
It should also be noted that the toy model does not alter the formula determining the present age of the Universe, which remains of a familiar form,

$$
t_{0}=\frac{1}{H_{0}} \int_{0}^{1} \frac{d x}{\left(1-\Omega_{0}+\Omega_{0} x^{-1}\right)^{1 / 2}} .
$$

[The suffix "0" in expression (178) and in the rest of the paper denotes the present value of any given quantity.] Since in the toy model $\Omega_{0}=1$, then the present age of the Universe is equal to $t_{0}=2 /\left(3 H_{0}\right)$. The latest research estimates the Hubble constant value to be $H_{0}=65 \pm 10 \mathrm{~km} \mathrm{~s}^{-1} \mathrm{Mpc}^{-1}$; see Ref. [18. Since the ages of the oldest globular clusters are estimated approximately to amount to $11.5 \pm 1.3 \mathrm{Gyr}$ [19], then the age of the Universe obtained from the formula (178) with $\Omega_{0}=1$ is not inconsistent with the observational data; see also Ref. [20].

\subsection{Cosmological density parameters}

This section contains considerations concerning defined in cosmology socalled total density parameter which is given as a sum of the mass density parameter $\Omega$ and a "vacuum" density parameter $\Omega_{\Lambda}$, the latter associated with the possible existence of a "macroscopic" cosmological constant $\Lambda$; we define the current value of the total cosmological density parameter as

$$
\Omega_{\text {total }} \equiv \Omega_{0}+\Omega_{\Lambda}
$$

where

$$
\Omega_{\Lambda} \equiv \frac{\Lambda c^{2}}{3 H_{0}^{2}} A=A \Omega_{\Lambda}^{\text {stand }} .
$$

The value of the parameter $\Omega_{\Lambda}$ has a significant impact on other cosmological quantities, like for instance the age of the Universe $t$ or the deceleration parameter $q$; note that in the considerations presented in section 5.1 we have assumed that $\Omega_{\Lambda}=0$.

\subsubsection{Cosmological mass density parameter $\Omega_{0}$}

First let us note that the vast majority of experimental cosmology measurements is related to the cosmological density parameter defined as in the standard model, i.e. to the quantity $\Omega_{0}^{\text {stand }}$. In order to determine its value it is not necessary - at least from the "experimental" point of view - to know 
the value of the factor $A$; see the formula (62).${ }^{18}$ Considerations presented in this subsection principally concern the parameter $\Omega_{0}^{\text {stand }}$, which depicts the density of matter occurring in the contemporary Universe, but neglects at the same time the existence of the extra spatial dimension, by omitting the factor $A$ in the definition of the quantity $\Omega_{0}$; see expressions (60)- (63).

On the grounds of the analysis of a number of observational data one might say that the cosmological density parameter $\Omega_{0}^{\text {stand }}$ takes the value from the interval $(0.01,1.1)$. The value of the quantity $\Omega_{0}^{\text {stand }}$ was estimated on the level $0.2-0.3$ based, among others, on the extensive studies of the dynamics of large astronomical objects, like clusters of galaxies; see, for instance, Refs. 21, 22, 23, 24 and references therein. One should ask, however, whether the value of the cosmological density parameter $\Omega_{0}^{\text {stand }} \in(0.2,0.3)$ determined in this manner is true, i.e. is it the value reflecting the real density of matter in the present Universe.

Let us note that in order to determine the value of the parameter $\Omega_{0}^{\text {stand }}$ by means of the analysis of the dynamics of large astronomical objects like mentioned clusters of galaxies, first of all, the mass(es) of investigated object(s) should be estimated. This, in turn, involves an appeal to the virial theorem, which has been described briefly in section 4.2 of this paper. However, for the determination of the mass of investigated object(s) - see Refs. [21, 22, 23, 24] and references therein - equation (29) of the standard Newtonian theory is used instead of equation (30) which results from the toy model. Consequently, the value of the object's mass so obtained is greater than that which would be determined within the framework of the toy model, i.e. applying Eq. (301). Thereby, the value of the cosmological density parameter $\Omega_{0}^{\text {stand }}$ obtained on the grounds of the discussed toy model can be considerably smaller than the value of $\Omega_{0}^{\text {stand }}$ determined by means of the standard theory which does not take into account the presence of the extra spatial dimension. Let us note, however, that the accurate determination of the value of the parameter $\Omega_{0}^{\text {stand }}$ based on the application of the virial theorem in terms of the toy model, seems to be very difficult in view of the fact that we do not know the value of the energy $U$ of the investigated object.

Hence, as far as we assume the possibility that the dark matter does

\footnotetext{
${ }^{18}$ Note that while experimentally estimating the value of the quantity $\Omega_{0}^{\text {stand }}$ applying the formula (62), the density of matter $\varrho_{0}$ is usually calculated as, roughly speaking, the quotient of the directly and/or indirectly detected mass by the (three-dimensional) volume of the space with $a=0$, within which (i.e. for $a=0$ ) as well as around which (i.e. for $a \neq 0)$ the detected mass is situated.
} 
not exist in the Universe in any significant amount, the real value of the parameter $\Omega_{0}^{\text {stand }}$ may be considered to be close to the value implied by the primordial baryon nucleosynthesis theory, which value is estimated to be $\Omega_{B}^{\text {stand }} \approx 0.020 \pm 0.007 h^{-2}$, where $h=H_{0} /\left(100 \mathrm{~km} \mathrm{~s}^{-1} \mathrm{Mpc}^{-1}\right)$; see Ref. 25] and references therein. This value is in accord with the recent estimations of the amount of the baryon matter in the present Universe, giving $0.007 \leq \Omega_{B}^{\text {stand }} \leq 0.041$ with a central value $\Omega_{B}^{\text {stand }} \approx 0.021[25]$; compare this result with the formula (68). Note that, in the case when the value of the parameter $\Omega_{0}^{\text {stand }}$ is so small that one has $\Omega_{0}^{\text {stand }} \approx \Omega_{B}^{\text {stand }}$, then the value of the deceleration parameter $q_{0}^{\text {stand }}=\Omega_{0}^{\text {stand }} / 2$ is very close to zero (i.e. of the order of $10^{-2}$ ). Such a small value of the deceleration parameter $q_{0}^{\text {stand }}$ would remain consistent with recently obtained experimental data [26].

We should also note that estimating the value of the cosmological density parameter $\Omega_{0}^{\text {stand }}$ to be of the order of $10^{-1}-10^{-2}$ allows one, according to the formula (66) , to estimate the quantity $A$ for the Universe to be of the order of $10^{1}-10^{2}$, which agrees with the order of the quantity $A$ which we assumed in section 5.1 of this paper. Thus, both the approaches: the theoretical one, investigated in section 5.1, and the experimental one, described in the present subsection, seem to remain consistent, as they give similar results.

It has already been mentioned that the determination of the real value of the cosmological density parameter $\Omega_{0}^{\text {stand }}$ in terms of the toy model seems to be very complicated. This is because of the fact that in many cases it depends on the knowledge of an a priori unknown value of the energy $U$, which may in principle vary for different objects. It looks, however, that the value of the mass of the investigated object(s) and then the value of the parameter $\Omega_{0}^{\text {stand }}$ can be determined in a relatively accurate manner by the extensive investigation of gravitational microlensing events, concerning both a luminous and a non-luminous matter. From section 4.1 of this paper we already know that there does not exist any additional light deflection caused by the existence of the extra spatial dimension. Therefore, in this case, the lack of knowledge of the parameter $U$ does not really matter.

And indeed, recently there have been undertaken numerous attempts at determining the mass of large astronomical objects that were based on the gravitational lensing effect; see Refs. [27, 28, 29, 30]. In several works, however, e.g. in Refs. 29, 30, a final determination of the cosmological density parameter $\Omega_{0}^{\text {stand }}$ involved the use of models assuming the existence of a sub- 
stantial amount of dark matter, ${ }^{19}$ e.g. by applying the virial theorem in the form of Eq. (29) rather than of Eq. (30). It seems that referring to the models assuming the existence of large amounts of dark matter in the Universe considerably limits the possibility of a completely objective - i.e. not depending on the applied method - determination of the value of the cosmological density parameter $\Omega_{0}^{\text {stand }}$.

Summarizing, it seems that the measurement of the mass of investigated $\operatorname{object}(\mathrm{s})$, which is necessary for the determination of the quantity $\Omega_{0}^{\text {stand }}$, should not be performed by means of the analysis of the investigated object's dynamics. The mass should rather be obtained, for example, by studying gravitational microlensing events, or from the analysis of the luminosity and mass-to-light ratio for investigated object(s). Among other interesting methods of the mass determination, used for instance to find masses of clusters of galaxies, is the analysis of the galaxy cluster X-ray integral temperature distribution function 32, 35.

Thus, in the context of this subsection, it seems to be reasonable to theoretically estimate the value of the quantity $\Omega_{0}^{\text {stand }}$ in such a manner that it incorporates the mass of the whole observable matter only, i.e. the mass of that matter which is concentrated within the range of the additional spatial dimension $a$, being subject to the direct perception of us, or rather of our measuring equipment; see the formula (68) and/or expressions (171) and (72) in section 5.1.

\subsubsection{Cosmological "vacuum" density parameter $\Omega_{\Lambda}$}

In this section we will discuss estimations regarding the values of the cosmological density parameters, obtained on the basis of observations of luminous sources known as type Ia supernovae; see Refs. [36, 37] and references therein. The analysis of the observational data concerning these objects leads to the conclusion that the values of both the cosmological parameters $\Omega_{0}$ and $\Omega_{\Lambda}$ are considerably greater than zero. On the basis of a number of measurements there were found, among others, the following values of the cosmological

\footnotetext{
${ }^{19}$ The models which assume the existence of a substantial amount of dark matter lead, among others, to the following relation $\sigma_{8}\left(\Omega_{0}^{\text {stand }}\right)^{0.5} \simeq 0.5$, linking together the value of the mass density parameter $\Omega_{0}^{\text {stand }}$ and the value of the amplitude of mass fluctuations, $\sigma_{8} ;$ see Refs. 31, 32, 33, 34.
} 
density parameters:

$$
\left(\Omega_{0}, \Omega_{\Lambda}\right) \approx(0.24,0.72),(0.00,0.48),(0.80,1.56),(0.72,1.48)
$$

see Ref. [36]. All of the above values were obtained by means of two different light-fitting methods: the first two by means of the so-called MLCS method, whereas the next two - with the use of the template fitting approach. In turn, in the recent paper of Perlmutter et al. 37, concerning the analysis of data on 42 high-redshift type Ia supernovae, there has been estimated a relationship between the cosmological parameters $\Omega_{0}$ and $\Omega_{\Lambda}$, which has the form

$$
0.8 \Omega_{0}-0.6 \Omega_{\Lambda} \approx-0.2 \pm 0.1 .
$$

A question arises here as to whether any of the above estimations approaches the real values of the cosmological density parameters. In an attempt to answer it, first let us note that the theoretical analysis of the observational data presented in Refs. [36, 37] is based on the so-called apparent magnitude-redshift relation; see Refs. [38, 39] and references therein.

Let $M$ and $m$ denote the absolute and apparent magnitude, respectively, at a given redshift $z$ for a luminous object. Results of measurements of both the quantities $M$ and $m$ depend on values of the cosmological density parameters $\Omega_{0}$ and $\Omega_{\Lambda}$ in a way described by the apparent magnitude-redshift relation which reads

$$
m(z)=M-5 \log H_{0}+25+5 \log \left[\mathcal{D}_{L}\left(z ; \Omega_{0}, \Omega_{\Lambda}\right)\right]+K,
$$

where $\log x \equiv \log _{10} x$. The quantity $K$ in the above formula denotes the socalled $K$-correction which appears in the above equation because the emitted and the detected photons coming from the receding object have different wavelengths; see Refs. [40, 41] and references therein. The quantity $\mathcal{D}_{L}$, where $\mathcal{D}_{L} \equiv d_{L} H_{0}$ and $d_{L}$ denotes the luminosity distance [1, is a function of the variables $z, \Omega_{0}$ and $\Omega_{\Lambda}$ [38; it should be emphasized that the function $\mathcal{D}_{L}$ does not depend on the value of the Hubble constant $H_{0}$. It can be easily shown that in the case of the toy model, under the assumption that $\Omega_{0}=1$ and $\Omega_{\Lambda}=0$, this function takes the following form,

$$
\mathcal{D}_{L}\left(z ; \Omega_{0}=1, \Omega_{\Lambda}=0\right)=c(1+z) \int_{0}^{z} \frac{d z^{\prime}}{\left(1+z^{\prime}\right)^{3 / 2}},
$$


whereas the general expression for $\mathcal{D}_{L}$ in the Friedmann standard model reads

$$
\begin{aligned}
\mathcal{D}_{L}\left(z ; \Omega_{0}, \Omega_{\Lambda}\right) & =\frac{c(1+z)}{\left|\Omega_{k}\right|^{1 / 2} \sin _{k}\left(\left|\Omega_{k}\right|^{1 / 2}\right.} \\
& \left.\times \int_{0}^{z} \frac{d z^{\prime}}{\left[\Omega_{0}\left(1+z^{\prime}\right)^{3}+\Omega_{k}\left(1+z^{\prime}\right)^{2}+\Omega_{\Lambda}\right]^{1 / 2}}\right),
\end{aligned}
$$

where $\Omega_{k} \equiv 1-\Omega_{0}-\Omega_{\Lambda}$ and $\sin _{k} x$ is equal to $\sinh x$ if $\Omega_{k}>0$, to $\sin x$ if $\Omega_{k}<0$, or to $x$ if $\Omega_{k}=0$. Note that, while deriving the formula (84), we have put $A=1$ for radial light ray(s) connecting the investigated supernova(e) and the observer. This is because, in the considered problem, the behaviour of the light particles' trajectories with respect to the dimension $a$ is not important, but only in the spacetime $a=0$; thus, the assumption that $A=1$ seems to be relevant here.

Moreover, there is usually defined a quantity called the "Hubble intercept",

$$
\mathcal{M} \equiv M-5 \log H_{0}+25 .
$$

To some extent, it can be measured directly, i.e. without knowing $H_{0}$, if we only know the apparent magnitude $m$ for objects showing relatively low redshifts $z$. In such a case, the relationship (83) takes the form

$$
m(z)=\mathcal{M}+5 \log (c z)+K .
$$

Thus, the quantity $\mathcal{M}$ can be obtained from low-redshift apparent magnitude measurements, i.e. from measurements of the apparent magnitudes and redshifts of low-redshift objects. Performing low-redshift measurements for objects of a similar type (e.g. type Ia supernovae) for the purpose of determining $\mathcal{M}$ and then carrying out measurements of the apparent magnitudes $m$ and redshifts $z$ for a number of distant, high-redshift objects of the same type, we can obtain the best-fit values of the cosmological density parameters $\Omega_{0}$ and $\Omega_{\Lambda}$ to solve Eq. (83); see Ref. 38.

It is known, however, that there exist perturbations on a spatially homogeneous and isotropic Universe. These perturbations can cause the value of the Hubble constant $H_{0}^{L}$ measured locally (redshift $z \leq 0.05$ ) to be different from the value of the global $(z>0.3)$ Hubble constant $H_{0}^{G}$. Note that in such a case, a definition of the directly measurable quantity $\mathcal{M}$ should take the following form,

$$
\mathcal{M} \equiv M-5 \log H_{0}^{L}+25
$$


Then, in the case when $H_{0}^{L} \neq H_{0}^{G}$, for the analysis of data concerning highredshift type Ia supernovae the following formula should be applied,

$$
\begin{aligned}
m(z) & =M-5 \log H_{0}^{G}+25+5 \log \left[\mathcal{D}_{L}\left(z ; \Omega_{0}=1, \Omega_{\Lambda}=0\right)\right]+K \\
& =\mathcal{M}+5 \log \frac{H_{0}^{L}}{H_{0}^{G}}+5 \log \left[\mathcal{D}_{L}\left(z ; \Omega_{0}=1, \Omega_{\Lambda}=0\right)\right]+K
\end{aligned}
$$

However, for this purpose equation (83) was used in Refs. [36, 37, which equation may be written in the following form,

$$
m(z)=\mathcal{M}+5 \log \left[\mathcal{D}_{L}\left(z ; \Omega_{0}^{a p p}, \Omega_{\Lambda}^{a p p}\right)\right]+K ;
$$

[the superscript app comes from the author of this paper.] It seems then that the quantities $\Omega_{0}^{a p p}$ and $\Omega_{\Lambda}^{a p p}$ are not the real cosmological parameters $\Omega_{0}$ and $\Omega_{\Lambda}$. If we compare Eq. (89) with Eq. (90), then it can be easily noticed that the quantities $\Omega_{0}^{a p p}$ and $\Omega_{\Lambda}^{a p p}$ depend in an essential way on the value of the quotient $H_{0}^{L} / H_{0}^{G}$, and also on the value of redshift $z$.

Deriving a formula for $\mathcal{D}_{L}$ in the case of the toy model, we have assumed that $\Omega_{0}=1$ and $\Omega_{\Lambda}=0$; see the equality (84). For the values of the apparent cosmological density parameters $\left(\Omega_{0}^{a p p}, \Omega_{\Lambda}^{a p p}\right)$ equal to $(0.24,0.72)$, $(0.00,0.48),(0.80,1.56)$ and $(0.72,1.48)[36]$ we obtain - from the comparison of Eq. (89) with Eq. (90), with taking into account the formulae (84) and (85) - the following values of the quotient $H_{0}^{L} / H_{0}^{G}: 1.219,1.223,1.270$ and 1.273, respectively. For the high-redshift supernovae investigated in Ref. 36] we have assumed here the average value of redshift $z_{a v} \approx 0.5$. It turns out that for substantial differences between the values of the apparent cosmological parameters $\Omega_{0}^{a p p}$ and $\Omega_{\Lambda}^{a p p}$ obtained by means of the two light-fitting methods, the values of the quotient $H_{0}^{L} / H_{0}^{G}$ remain almost the same for each of the light-fitting methods separately. Therefore, one should consider the hypothesis that the real values of the cosmological parameters $\Omega_{0}$ and $\Omega_{\Lambda}$ are close to 1 and 0 , respectively - as predicted by the toy model, whereas for objects with a small redshift $z$ the value of the locally measured Hubble constant $H_{0}^{L}$ is slightly greater than its global value $H_{0}^{G}$. To some extent, this effect may occur due to the peculiar streaming motion in our neighbourhood which is toward the so-called Great Attractor, situated at the redshift $z \sim 0.02$; see Ref. [42] and/or Fig. 5.4 in Ref. [1].

Now let us discuss results presented in Ref. [37. If we take the average redshift for the investigated objects to be equal to $z_{a v} \approx 0.5$, then we can determine the value of the quotient $H_{0}^{L} / H_{0}^{G}$ for different values of 
the parameters $\Omega_{0}^{a p p}$ and $\Omega_{\Lambda}^{a p p}$ which satisfy Eq. (82). For instance, for $\left(\Omega_{0}^{a p p}, \Omega_{\Lambda}^{a p p}\right)=(0 \pm 5 / 80,1 / 3 \pm 1 / 12)$ we obtain $H_{0}^{L} / H_{0}^{G}=1.193 \pm 0.029$. The value of the quotient $H_{0}^{L} / H_{0}^{G}$ grows very slowly with increasing values of the cosmological parameters $\Omega_{0}^{a p p}$ and $\Omega_{\Lambda}^{a p p}$, becoming equal e.g. to $H_{0}^{L} / H_{0}^{G}=1.241 \pm 0.035$ for $\left(\Omega_{0}^{a p p}, \Omega_{\Lambda}^{a p p}\right)=(1 \pm 5 / 80,5 / 3 \pm 1 / 12)$. Such a small difference between the values of the quotient $H_{0}^{L} / H_{0}^{G}$ for considerably varying values of the apparent cosmological density parameters $\Omega_{0}^{a p p}$ and $\Omega_{\Lambda}^{a p p}$ which satisfy empirically obtained Eq. (82), seems to confirm the hypothesis that the real values of the cosmological density parameters $\Omega_{0}$ and $\Omega_{\Lambda}$ are close to unity and zero, respectively. The value of the local Hubble constant $H_{0}^{L}$ for objects with small redshifts $(z \leq 0.05)$ whereas is somewhat greater than the value of the global Hubble constant $H_{0}^{G}$.

However, any binding verification of this hypothesis is not easy, especially if one keeps in mind the difficulties in independently of one another determining the four quantities: $H_{0}^{L}, H_{0}^{G}, \Omega_{0}$ and $\Omega_{\Lambda}$. In the case of the analysis of the observational data that concern type Ia supernovae, usually two of the above quantities had been a priori fixed, which in the next step allowed one the determination of the remaining two other parameters. For instance, in Refs. [36, 37] it had been assumed that $H_{0}^{L}=H_{0}^{G}$ and the attempts were made to determine the values of $\Omega_{0}$ and $\Omega_{\Lambda}$ or - to be more precise - of $\Omega_{0}^{\text {stand }}$ and $\Omega_{\Lambda}^{\text {stand }}$. Alternatively, in Refs. [40, 41] the quantities $\Omega_{0}$ and $\Omega_{\Lambda}$ were left as free (changing) parameters and the authors instead tried to determine a value of the quotient $H_{0}^{L} / H_{0}^{G}$, each time for some fixed values of the quantities $\Omega_{0}$ and $\Omega_{\Lambda}$, i.e. as a (two-variable) function of both the quantities $\Omega_{0}$ and $\Omega_{\Lambda}$.

Moreover, let us note that - in general - different observed "local supernovae calibrators" (i.e. low-redshift supernovae) may lie within different cosmological local or sub-local flows (which is, of course, rather improbable for a set of supernovae situated within a sufficiently small region of space). This could mean that for different local supernovae the quantity $H_{0}^{L}$ might have varying values. On the other hand, it cannot be ruled out that also some of the observed high- $z$ supernovae lie within certain local cosmological flows; of course, values of the Hubble constant for such objects might differ from the value of the global Hubble constant $H_{0}^{G}$. It then seems that, in order to come to a conclusion concerning the real values of the parameters $\Omega_{0}$ and $\Omega_{\Lambda}$, many more data on the spatial distribution of the value of the Hubble constant are needed.

Despite all the above objections and difficulties in determining the real 
values of the cosmological parameters $\Omega_{0}$ and $\Omega_{\Lambda}$ based on the supernovae observational data, it should be stated that the method presented in this subsection is - if one assumes the existence of the extra spatial dimension much more promising than studying of the dynamics of large astronomical objects. It is caused by the fact that in none of the equations and formulae applied in this subsection does there seem to be any dependence upon the a priori unknown quantity $U$, whose occurrence complicates considerably attempts to determine the real values of the cosmological density parameters in the case of analysing the dynamics of large astronomical objects by means of the virial theorem.

\subsubsection{Fluctuations of cosmic microwave background radiation}

Assuming that $\Omega_{0}=1.0$, we can estimate the value of the cosmological density parameter $\Omega_{0}^{\text {stand }}$ defined as in the standard model, which is connected to the value of the quantity $\Omega_{0}$ by the relation (63). Taking into account that for the present Universe as a whole the value of the factor $A$ can be of the order of 10 , one can suppose that $\Omega_{0}^{\text {stand }} \sim 10^{-1}$. Could the above statement be an important fact that might help to explain some discrepancies between various observational data as well as between some experimental and theoretical results (the latter, for instance, coming from a computer simulation)? According to the latest reports, the value of the cosmological mass density parameter close to 0.1 (see section 5.2.1), on the assumption that $\Omega_{\Lambda}=0$ (see section 5.2.2), is not consistent with the observed fluctuations of the cosmic microwave background radiation (CMBR).

The value and shape of the CMBR fluctuations, and in effect also of primordial fluctuations in the density of matter, are reflected in the angular power spectrum of CMBR. As it turns out, the position of the first acoustic (Doppler) peak at the Legendre multipole in the angular power spectrum of the temperature fluctuations of CMBR is almost exclusively determined by the value of the cosmological density parameter $\Omega_{0}$, and varies as

$$
\ell_{\text {peak }} \simeq \frac{200}{\sqrt{\Omega_{0}}} ;
$$

this occurs because the angular scale $\ell_{\text {peak }}$ of the main peak reflects the size of the horizon at last scattering of the CMBR photons; see Ref. [43] and references therein as well as Ref. [1]. It should be stressed that the quantity $\Omega_{0}$ in the formula (911) corresponds in the toy model to the cosmological mass 
density parameter defined in expression (61), and not to the cosmological mass density parameter $\Omega_{0}^{\text {stand }}$ defined as in the standard model and given by the relation $\Omega_{0}^{\text {stand }}=A^{-1} \Omega_{0}$; roughly speaking, this occurs, since the factor $\dot{R} / R$ - which enters the formula for the optical depth to the surface of the Thomson ("last") scattering by free electrons [1] - is proportional to $\Omega$, and not to $\Omega^{\text {stand }}$; see Eq. (43) and expressions (60), (62).

Based on studies of the power spectrum of CMBR, it can be stated that the observed fluctuations of CMBR are too small to have been produced in an open Universe with small values of $\Omega_{0}$ like, for instance, $\Omega_{0}=0.3$. It turns out, that only values $\Omega_{0}>0.4$ remain consistent with the degree of fluctuations of the cosmic microwave background radiation as well as with the recent estimations concerning the Hubble constant value [43.

A new limit on the value of the cosmological parameter $\Omega_{0}$ obtained in Ref. [43] reads $\Omega_{0}=0.7_{-0.5}^{+0.8}$, which remains consistent with our theoretical result $\Omega_{0}=1$; see the formula (65). The most recent Boomerang experiment [44] indicates that $\ell_{\text {peak }}=(197 \pm 6)$, which strongly favours a flat Universe, i.e. is a significant confirmation of the hypothesis that $\Omega_{0} \approx 1$. Thus, it seems that within the framework of the toy model, the currently observed value of the CMBR fluctuations remains consistent with the notion of the toy-model universe in which the value of the cosmological mass density parameter $\Omega_{0}$ is equal to one.

\subsubsection{Conclusions}

We can say that the toy model introduces quite significant corrections to definitions of the most important cosmological parameters, simultaneously introducing considerable revision of the methodology of the experimental determination of their values. This may cause, for instance, that the values of the cosmological density parameters - obtained by means of different methods from different observational data - will be consistent with each other, thereby maintaining the self-consistency of the model.

In particular, we should draw our attention to the two kinds of measurements:

i) The measured (or calculated with the use of other measured quantities) value of the cosmological mass density parameter is considerably smaller than one; it appears that in such a case the measured (or calculated) quantity could be the cosmological density parameter $\Omega_{0}^{\text {stand }}$ defined as in the standard model; see section 5.2.1. 
ii) The measured (or calculated from other measured quantities) value of the cosmological mass density parameter is close to one; it turns out that the measured (or calculated) quantity corresponds to the cosmological density parameter $\Omega_{0}$ defined as in the toy model, the value of which, according to the formula (65), is equal to one; see section 5.2.3.

\subsection{Early times of the Universe}

The last feature of the toy-model universe that we would like to address in this section is its possible lacking of the initial singularity, i.e. the absence of the singularity at the moment $t=0 .\left({ }^{20}\right)$ First, we observe that as the macrospace $\mathcal{R}^{3}$ of the Universe is flat - see the formula (65) - and then infinite, so it existed also for $t \leq 0$. We then assume that $R(t \leq 0)=$ const $\equiv R_{s}>0$. In such a situation, the spacetime described by the metric (36) takes the form of the Cartesian (direct) product of the covering surface $\mathcal{R}^{1} \times \mathcal{R}^{1}$ for the antide Sitter two-dimensional universe times the static flat three-dimensional macrospace $\mathcal{R}^{3}$, and we have

$$
\begin{aligned}
d s^{2}= & \left(1+|\lambda| a^{2}\right) c^{2} d t^{2}-\left(1+|\lambda| a^{2}\right)^{-1} d a^{2} \\
& -R_{s}^{2}\left(d r^{2}+r^{2} d \theta^{2}+r^{2} \sin ^{2} \theta d \varphi^{2}\right) .
\end{aligned}
$$

It cannot be ruled out, however, that the primordial toy universe (before the Big Bang, i.e. for $t<0$ ) was actually an anti-de Sitter universe of the form $\mathcal{S}^{1} \times \mathcal{R}^{1} \times \mathcal{R}^{3}$, with closed time-like (or null for radiation) curves, the whole history of which used to repeat every $T_{l} \sim 10^{-43}$ seconds. Such a universe may be described by the metric

$$
d s^{2}=c^{2} d t^{2}-\exp (i 2 \sqrt{|\lambda|} c t) d a^{2}-R_{s}^{2}\left(d r^{2}+r^{2} d \theta^{2}+r^{2} \sin ^{2} \theta d \varphi^{2}\right)
$$

which we obtain after a purely formal (complex) coordinate transformation performed for the metric (92); in the case of the metric (93) the existence of the closed time-like (or null for radiation) curves in the anti-de Sitter universe is especially easy to notice. Note that the value of the zero-point energy (of the vacuum) in such a spacetime was exactly the same as in the present toy universe; see section 2.

\footnotetext{
${ }^{20}$ Note that some non-singular models of the Universe are also discussed, for instance, in Refs. 45, 46, 47, 48, 49, 50.
} 
We will now examine the features of the early Universe in a more precise manner. Let us first note that the Ricci scalar curvature $R_{\mu}^{\mu}$ - present in expression for the action for the Einstein field equation - provides the Lagrangian density for the spacetime geometry represented by the metric tensor [1. Let us suppose the Lagrangian density - so also the Ricci scalar to have remained a continuous quantity at the moment of the Big Bang (i.e. for $t=0)$; of course, we assume here that the quantity $\operatorname{det}\left(g_{\mu \nu}\right)$ is continuous during the whole history of the Universe. The Ricci scalar should then fulfil the following equation,

$$
R_{\mu}^{\mu}\left(t \rightarrow 0^{-}\right)=R_{\mu}^{\mu}\left(t \rightarrow 0^{+}\right) .
$$

We assume that the Universe before, after, and at the moment of the Big Bang was described by the Einstein equation (11), with the stress-energy tensor given by expressions (37) -(39) and (2). Moreover, we assume that the stress-energy tensor $\widehat{T}_{\mu \nu}$, given by the formulae (38) and (39), was equal to zero before the Big Bang. We will prove later that this assumption may be valid. From Eq. (94) we then obtain the following relationship between the density $\varrho$ of matter, or of the radiation energy and its pressures $p$ and $\widetilde{p}$,

$$
\widetilde{p}+3 p=c^{2} \varrho .
$$

Combining the above equation with Eq. (52) which is expected to be fulfilled also in the limit $t \rightarrow 0^{+}$, gives

$$
\begin{aligned}
& p=\frac{c^{2}}{3} \varrho \\
& \widetilde{p}=0 .
\end{aligned}
$$

Thus, in the toy model, the Universe at the moment of the Big Bang was filled with a perfect relativistic fluid, or radiation. The question arises as to what was the value of the energy density of the fluid at the moment $t=0$, i.e. what was the initial condition for the density of the radiation energy in the Universe. To answer this question, let us imagine that the Universe before the Big Bang was of the form of the anti-de Sitter spacetime $\mathcal{S}^{1} \times \mathcal{R}^{1} \times \mathcal{R}^{3}$. We know that the anti-de Sitter spacetime $\mathcal{S}^{1} \times \mathcal{R}^{1}$ contains "global" closed time-like or null curves; see sections 3 and 4.2 of this paper. Let us assume that such a universe was filled with the radiation whose particles had a period of oscillation

$$
T_{l}=\frac{2 \pi}{c \sqrt{|\lambda|}}
$$


so they retraced their own life histories after each lapse of the period $T_{l}$ of the coordinate time $t$. Thus, the geodesic lines of the radiation particles (quanta) were closed null curves, so the radiation was "frozen", or "fixed" in time $t$ in the anti-de Sitter spacetime; see Fig. 2. Note that the distance covered - during the coordinate time equal to the period of oscillation $T_{l}-$ by the radiation particles (quanta) in the "macroscopic" space $\mathcal{R}^{3}$ was equal to $L=c T_{l}$, which quantity we can call a "wavelength" of oscillation. The "frozen" radiation in the macrospace $\mathcal{R}^{3}$ might be described, or imagined in such a way that each quantum of the radiation covered the distance $L$, equal to its (one) wavelength, during the coordinate time $T_{l}$, equal to the period of its oscillation; note that, due to the existence of the closed curves in the investigated anti-de Sitter spacetime, the time $T_{l}$ may be regarded as the whole period, or interval of time $t$ which elapsed before the Big Bang; see Fig. 2. It should be added here that in the two above sentences as well as in the remaining part of this paper, under the terms "macroscopic" space, or macrospace $\mathcal{R}^{3}$ we understand mainly the three-dimensional space given by the expression $a \approx 0$.

What happened at the moment of the Big Bang? It seems that the anti-de Sitter two-dimensional spacetime $\mathcal{S}^{1} \times \mathcal{R}^{1}$ should have undergone a phase transition into the covering surface $\mathcal{R}^{1} \times \mathcal{R}^{1}$ of the anti-de Sitter spacetime, described by the metric (7), so the coordinate time $t$ was released to elapse monotonically and not periodically as before the Big Bang. This caused that the radiation started to give a (non-vanishing) contribution to the stress-energy tensor $\widetilde{T}_{\mu \nu}$, in the form represented by the tensor $\widehat{T}_{\mu \nu}$; see the formula (37). This, in turn, gave the beginning to the expansion of the Universe, i.e. of the flat three-dimensional space $\mathcal{R}^{3}$, according to Eqs. (40)(42). Simultaneously, the radiation, confined before the Big Bang within the anti-de Sitter two-dimensional spacetime and forming "frozen waves" in the macrospace $\mathcal{R}^{3}$, was released into the expanding three-dimensional "macroscopic" space. The Big Bang event occurring in the toy model could then be described as the following "spacetime" - or rather only "time" transition,

$$
\mathcal{S}^{1} \times \mathcal{R}^{1} \times \mathcal{R}^{3} \stackrel{t=0}{\longrightarrow} \mathcal{R}^{1} \times \mathcal{R}^{1} \times\left(\mathcal{R}^{3}\right)_{\text {expand }} .
$$

What was the energy density of the radiation at the moment of the Big Bang? The energy of one quantum of the radiation confined before the Big 
Bang within the anti-de Sitter two-dimensional spacetime is easy to calculate,

$$
E=\hbar \frac{2 \pi}{T_{l}}=\hbar \sqrt{|\lambda|} c
$$

it is, in fact, equal to the Planck energy as defined in section 4.2. We have to multiply the energy $E$ by a factor of 3, if we want to take into account the three orthogonal to each other as well as independent of - or "noninterfering" with - one another spatial modes of the radiation, as the latter filled the three-dimensional macrospace $\mathcal{R}^{3}$. Furthermore, the so-obtained number should then be multiplied by a factor of 4 , since the existence of any trajectory $(t, a, x, y, z)[\gamma]$ of the radiation quantum in the spacetime described by the metric (92) or (93) implies, for e.g. $y, z=$ const, the existence of the trajectories: $(t,-a, x, y, z)[\gamma]$ and $(t, a,-x, y, z)[\gamma]$ as well as $(t,-a,-x, y, z)[\gamma]$; see also the formula (13) for comparison. Note, however, that here we do not take into account the symmetry $\gamma \leftrightarrow-\gamma$ of the investigated solution to the Einstein equation (11), as both kinds of the trajectories: $(\cdot)[\gamma]$ and $(\cdot)[-\gamma]=-(\cdot)[\gamma]$, remain the same in the spacetime of the toy model; it does not, of course, exclude the existence of antiparticles - see the last sentence of the discussion concerning antiparticles in section 3.1 of this paper. Thus, bearing in mind the above discussion concerning the closed null curves and the retracing of the life history by the radiation quanta, one can assume that (for $a=0$ ) the (twelve) quanta possessing the energy $12 E$ occupied the region $L \times L \times L$ of the macrospace $\mathcal{R}^{3}$. If we then conventionally define the energy density $u$ as a quotient of the energy concentrated in some region of the (three-dimensional) space by the volume of this region, then we will obtain the energy density of the radiation at the moment of the Big Bang,

$$
u(t \leq 0, a=0)=\frac{12 E}{L^{3}}=\frac{3 \hbar \lambda^{2} c}{2 \pi^{3}} \cong 2.242 \times 10^{112} \mathrm{~kg} \mathrm{~s}^{-2} \mathrm{~m}^{-1} .
$$

As the macrospace $\mathcal{R}^{3}$ was/is flat during the whole history of the toy universe, so consequently one has $\Omega=1$, then we obtain

$$
H\left(t \rightarrow 0^{+}\right)=\left[\frac{8 \pi G}{3 c^{2}} u(t \leq 0, a=0)\right]^{1 / 2} \cong 1.181 \times 10^{43} \mathrm{~s}^{-1} .
$$

Note that after the Big Bang (occurring at the moment $t=t_{s}=0$ ) the energy density of the radiation should, according to Eqs. (43), (53) and (49), 
have been decreasing following the formula

$$
u(t, a)=\left[2 X\left(t-t_{s}\right)+\left(\frac{u_{s}}{A}\right)^{-1 / 2}\right]^{-2}
$$

where $X \equiv\left(-\kappa c^{2} A / 3\right)^{1 / 2}$ and $u_{s} \equiv u\left(t=t_{s}, a=0\right)$. It is easy to see - compare, for instance, the formulae (92) and (6) as the two solutions to formally the same Einstein equation with the same stress-energy tensor that the initial condition for the scale factor $R$ may be e.g. expressed as follows,

$$
R_{s} \equiv R(t \leq 0)=1 \mathrm{~m},
$$

which allows one to determine the value of the integration constant resulting from the continuity equation for radiation, $u R^{4}=u_{s} R_{s}^{4}=$ const, as well as to obtain the value of the quantity $\dot{R}\left(t \rightarrow 0^{+}\right)$, from Eq. (43). We should note that if we assume the cosmic scale factor $R$ to be an "order parameter" for the toy-model universe, then we can recognize the process described by expression (99) as a phase transition of the first order, since the quantity $R$ remained continuous at the moment of the Big Bang, whereas its derivative $\dot{R}$ was not continuous at the time $t=0$, as one has $\dot{R}\left(t \rightarrow 0^{-}\right)=0 \neq \dot{R}\left(t \rightarrow 0^{+}\right)$; of course, this happened because exactly at the moment of the Big Bang the radiation filling the toy universe just started to give a (non-vanishing) contribution to the stress-energy tensor $\widetilde{T}_{\mu \nu}$.

Now we will try to show that the stress-energy tensor $\widehat{T}_{\mu \nu}$ was equal to zero before the Big Bang. To this aim let us assume that the smallest element of the fluid ("frozen" radiation), which the toy universe before the Big Bang was filled with, was - in the macrospace $\mathcal{R}^{3}$ - the cube of the sizes $L \times L \times L .\left({ }^{21}\right)$ The cube was filled with the four - symmetrical with respect to the four hypersurfaces $a=0$ and $x, y, z=$ const as well as noninterfering with each other - sets of the three independent of and orthogonal to one another spatial modes of the "frozen" radiation, each of the wavelength $L=c T_{l}$. The sizes of the (hyper)cube in the directions $t$ and $a$ were equal to $T_{l} \equiv L / c$ and $L / \pi$ [see the formula (19)], respectively. We should now recall the general definition of a stress-energy tensor $T^{\mu \nu}$, which determines the element $T^{\mu \nu}$ for a particular $\mu$ and $\nu$ as the flux density of the $\mu$-component

\footnotetext{
${ }^{21}$ One could not imagine a smaller element of the fluid than that of the sizes $L \times L \times L$, since each of the three sizes of the smallest fluid element should contain at least one full length of the oscillations of the radiation quantum, i.e. exactly the quantity $L$.
} 
of momentum in the direction $\nu$, i.e. through the surface with a constant value of the coordinate $x^{\nu}$, orthogonally to it. It is then clear that the flux density of any component $\mu=0, \ldots, 4$ of momentum through any of the surfaces - with constant values of the coordinates $x^{\nu}$ (for all $\left.\nu=0, \ldots, 4\right)-$ of the hyperbox $c T_{l} \times L / \pi \times L \times L \times L$ was actually equal to zero before the Big Bang. ${ }^{22}$ This happened due to the fact that before the Big Bang the geodesic lines of the radiation quanta were the closed null curves with the closure times (or the oscillation periods) all equal to $T_{l}$, so none of the surfaces of the considered hyperbox was crossed by the radiation quanta during the period of time $T_{l}$, and/so the time $T_{l}$ may actually be regarded as the whole period, or interval of time $t$ which elapsed before the Big Bang. Let us note that the above considerations seem to be true not only for the element $c T_{l} \times L / \pi \times L \times L \times L$ of the fluid filling the toy universe before the Big Bang, but also remain valid for any multiplication of this element, of the form $n_{1} c T_{l} \times n_{2} L / \pi \times n_{3} L \times n_{4} L \times n_{5} L$, where all $n_{1}, \ldots, n_{5}$ are natural numbers.

What happened later? After the lapse of a some time after the Big Bang, the perfect relativistic fluid, or radiation - expanding within/together with the three-dimensional "macroscopic" space $\mathcal{R}^{3}$ - started to convert into "ordinary" matter, for which the relative ratio $p / u$ decreases from the value $1 / 3$ to 0 , and thus, according to Eq. (52), the pressure $\widetilde{p}$ along the extra spatial dimension $a$ decreases from 0 to $-u / 2$.

It is important to observe that, apart from the attempt to answer the question about the initial conditions at the moment of the Big Bang, the toy model seems to enable us also to solve the problem of the large-scale spatial homogeneity and isotropy of the present Universe. Namely, the toymodel universe existed already before the Big Bang and each (separate) region $L \times L \times L$ of the "macroscopic" (flat) three-dimensional space $\mathcal{R}^{3}$ before the Big Bang was filled with the radiation of the same "initial" energy $12 E$, where the quantity $E$ is given by the formula (100). We may then understand that the energy density of the radiation filling the early Universe was highly isotropic already at the moment of the Big Bang. The possible fluctuations in the density of energy/matter, which started to grow some time after the Big

\footnotetext{
${ }^{22}$ It is of particular interest to observe that the above conclusion holds also for the surfaces of the considered hyperbox, which are given by $a=$ const, or $a= \pm L /(2 \pi)$, and $t=$ const $\pm n T_{l}$, where $n \in \mathcal{N} \cup\{0\}$.
} 
Bang, could have been caused by the fact that the energy $U$ at the moment of the Big Bang could have varied for different quanta of the radiation.

\section{Summary}

In this paper we have investigated the toy model which originates from general relativity. It provides an extension of the dimensionality of spacetime, with an additional dimension of space macroscopically unobservable. The model attempts to give a solution to the problem of the cosmological constant.

It turns out that the toy model introduces no corrections to most predictions of the "standard" general relativity regarding, among others, the so-called "five tests of general relativity". However, it seems that the toy model could provide an explanation to the flatness of circular velocity curves of spiral galaxies without introducing any dark matter.

The toy model introduces certain changes into cosmology, altering the definition of the critical density of matter. Consequently, it also changes - as compared with the Friedmann standard model - the values assumed by other cosmological parameters. Due to the introduction of the additional spatial dimension, data concerning the present value of the Universe's mass density obtained, for instance, from observations of distant supernovae seem to be consistent with other measurements, such as those regarding the temperature fluctuations of the cosmic microwave background radiation. Finally, no initial singularity is present in the proposed model.

\section{Acknowledgments}

The author is most grateful to Alexander von Humboldt-Stiftung for support and for making possible his research stay in Germany, where studies on the subject discussed in this paper were started. He is also indebted to Professor W. Kopczyński for his critical remarks regarding the very preliminary version of sections 2 and 3 of this essay.

\section{References}

[1] P. J. E. Peebles, Principles of Physical Cosmology (Princeton University Press, Princeton, 1993). 
[2] S. Weinberg, Rev. Mod. Phys. 61, 1 (1989).

[3] S. W. Hawking and G. F. R. Ellis, The Large Scale Structure of SpaceTime (Cambridge University Press, Cambridge, 1973).

[4] W. Thirring, Lehrbuch der Mathematischen Physik (Springer-Verlag, Wien, 1977).

[5] V. A. Rubakov and M. E. Shaposhnikov, Phys. Lett. 125B, 136 (1983).

[6] A. Einstein, B. Podolsky and N. Rosen, Phys. Rev. 47, 777 (1935).

[7] J.-M. Souriau, Nuovo Cimento 30, 565 (1963).

[8] S. D. Unwin, Phys. Lett. 103B, 18 (1981).

[9] C. W. Misner, K. S. Thorne and J. A. Wheeler, Gravitation (Freeman, San Francisco, 1973).

[10] J. Binney and S. Tremaine, Galactic Dynamics (Princeton University Press, Princeton, 1987).

[11] M. Milgrom, Astrophys. J. 270, 365 (1983).

[12] J. Bekenstein and M. Milgrom, Astrophys. J. 286, 7 (1984).

[13] H. Böhringer, Clusters of galaxies. In Proceedings of the 17th Texas Symposium on Relativistic Astrophysics and Cosmology, eds. H. Böhringer, J. Trümper and G. E. Morfill (The New York Academy of Sciences, New York, 1995).

[14] H. Böhringer, D. M. Neumann, S. Schindler and R. C. Kraan-Korteweg, Astrophys. J. 467, 168 (1996).

[15] P. D. Mannheim, Astrophys J. 419, 150 (1993).

[16] P. D. Mannheim, Astrophys. J. 479, 659 (1997).

[17] W. L. Freedman and M. S. Turner, Rev. Mod. Phys. 75, 1433 (2003).

[18] W. L. Freedman, J. R. Mould, R. C. Kennicutt Jr. and B. F. Madore, The Hubble Space Telescope key project to measure the Hubble constant. In IAU Symposium No. 183: Cosmological Parameters and the Evolution of the Universe, ed. K. Sato (Kluwer, Dordrecht, 1999). 
[19] B. Chaboyer, P. Demarque, P. J. Kernan and L. M. Krauss, Astrophys. J. 494, 96 (1998).

[20] B. Chaboyer, Phys. Rep. 307, 23 (1998).

[21] R. G. Carlberg, H. K. C. Yee, E. Ellingson, R. Abraham, P. Gravel, S. Morris and C. J. Pritchet, Astrophys. J. 462, 32 (1996).

[22] N. A. Bahcall, X. Fan and R. Cen, Astrophys. J. 485, L53 (1997).

[23] X. Fan, N. A. Bahcall and R. Cen, Astrophys. J. 490, L123 (1997).

[24] M. Donahue, G. M. Voit, I. M. Gioia, G. Luppino, J. P. Hughes and J. T. Stocke, Astrophys. J. 502, 550 (1998).

[25] M. Fukugita, C. J. Hogan and P. J. E. Peebles, Astrophys. J. 503, 518 (1998).

[26] K. Rines, W. Forman, U. Pen, C. Jones and R. Burg, Astrophys. J. 517, 70 (1999).

[27] I. Smail, R. S. Ellis, M. J. Fitchett and A. C. Edge, Mon. Not. R. Astron. Soc. 273, 277 (1995).

[28] G. A. Luppino and N. Kaiser, Astrophys. J. 475, 20 (1997).

[29] N. A. Bahcall and X. Fan, Astrophys. J. 504, 1 (1998).

[30] J. Hjorth, J. Oukbir and E. van Kampen, Mon. Not. R. Astron. Soc. 298, L1 (1998).

[31] V. R. Eke, S. Cole and C. S. Frenk, Mon. Not. R. Astron. Soc. 282, 263 (1996).

[32] P. T. P. Viana and A. R. Liddle, Mon. Not. R. Astron. Soc. 281, 323 (1996).

[33] U.-L. Pen, Astrophys. J. 498, 60 (1998).

[34] T. Kitayama and Y. Suto, Astrophys. J. 469, 480 (1996).

[35] P. T. P. Viana and A. R. Liddle, Mon. Not. R. Astron. Soc. 303, 535 (1999). 
[36] A. G. Riess et al., Astronom. J. 116, 1009 (1998).

[37] S. Perlmutter et al., Astrophys. J. 517, 565 (1999).

[38] A. Goobar and S. Perlmutter, Astrophys. J. 450, 14 (1995).

[39] S. Perlmutter et al., Astrophys. J. 483, 565 (1997).

[40] A. Kim et al., K corrections for type Ia supernovae and a test for spatial variation of the Hubble constant (The Supernova Cosmology Project: II). In Thermonuclear Supernovae (NATO ASI), eds. R. Canal, P. RuizLaPuente and J. Isern (Kluwer, Dordrecht, 1997).

[41] A. G. Kim et al., Astrophys. J. 476, L63 (1997).

[42] J. R. Mould et al., Astrophys. J. 383, 467 (1991).

[43] S. Hancock, G. Rocha, A. N. Lasenby and C. M. Gutiérrez, Mon. Not. R. Astron. Soc. 294, L1 (1998).

[44] P. de Bernardis et al., Nature 404, 955 (2000).

[45] M. Israelit and N. Rosen, Astrophys. J. 342, 627 (1989).

[46] H. J. Blome and W. Priester, Astron. Astrophys. 250, 43 (1991).

[47] S. P. Starkovich and F. I. Cooperstock, Astrophys. J. 398, 1 (1992).

[48] S. S. Bayin, F. I. Cooperstock and V. Faraoni, Astrophys. J. 428, 439 (1994).

[49] J. M. Overduin and F. I. Cooperstock, Phys. Rev. D 58, 043506 (1998).

[50] E. Rebhan, Astron. Astrophys. 353, 1 (2000). 


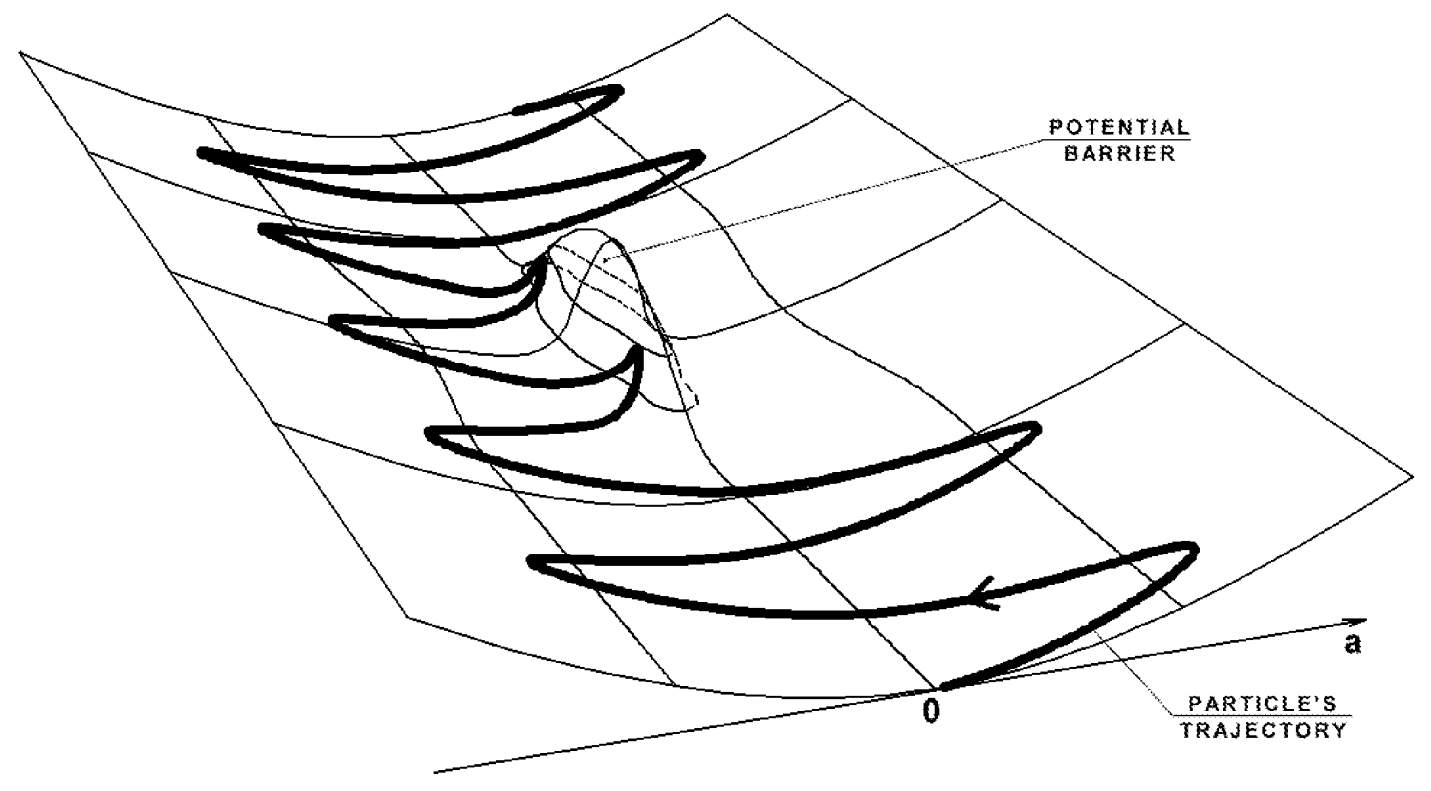

Figure 1. A particle, moving with a uniform rectilinear motion with respect to the spacetime $a=0$, simultaneously oscillates in the additional fourth spatial dimension $a$. The particle encounters an obstacle - a potential barrier higher than its energy $E$. However, because of the oscillations in the fourth spatial dimension, the particle can surmount the barrier, by simply circumventing it. The nature of this phenomenon is purely geometric and thus extremely simple. We can see here an analogy to a tunnelling through the potential barrier, the phenomenon known from quantum mechanics and still puzzling - as far as its nature is concerned. According to the corpuscular interpretation of quantum mechanics, at the moment of the tunnelling the particle temporarily "vanishes" to appear subsequently on the opposite side of the barrier. In the toy model presented in this paper, the particle can "vanish" in the extra spatial dimension, which may allow it to circumvent the potential barrier.

Let us note that the particle can cross the potential barrier (or rather circumvent it) as well as be reflected. Naturally, the answer to the question of whether a particle will cross (circumvent) the potential barrier or whether it will rebound, depends on a number of factors such as: the values of the particle's energies $U$ and $E$, the barrier sizes as well as the barrier shape with respect to the extra spatial dimension a. Note. The figure is merely demonstrative and does not preserve proportions between various quantities characterizing both the particle and the potential barrier. 


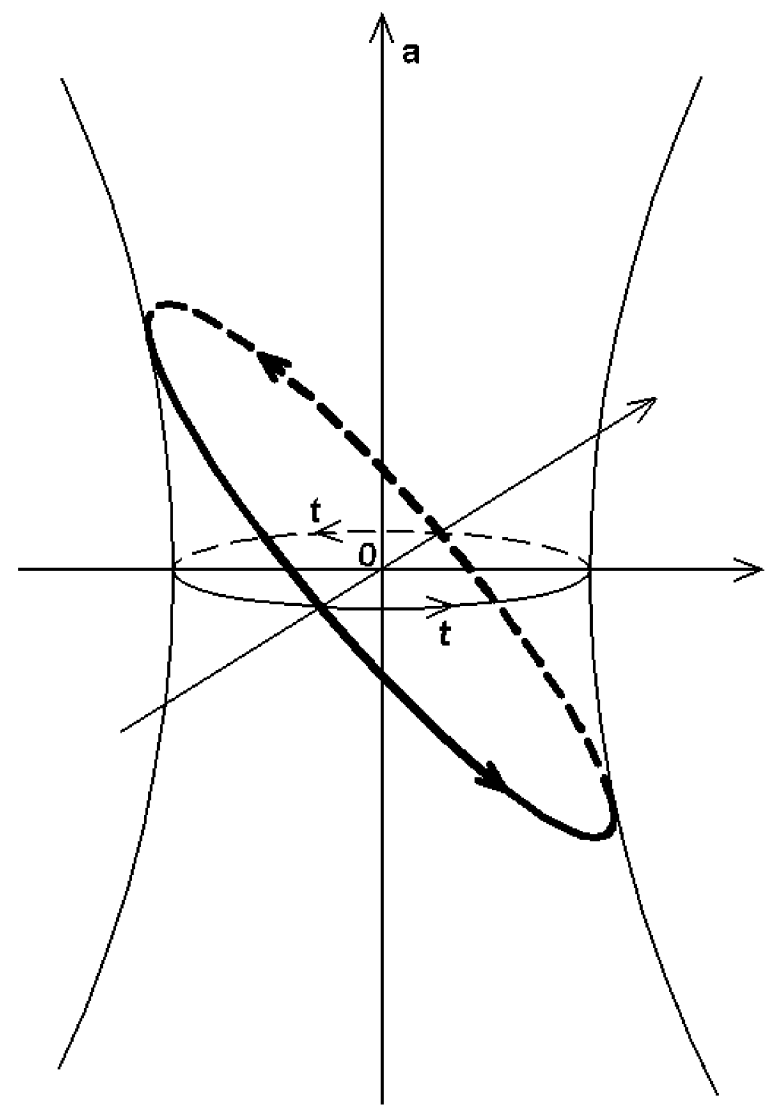

Figure 2. The toy model predicts that the Universe before the Big Bang was of the form of the anti-de Sitter spacetime $\mathcal{S}^{1} \times \mathcal{R}^{1} \times \mathcal{R}^{3}$ filled with the radiation quanta, each of energy $E$ equal to the Planck energy $E_{P l}$. The geodesic line(s) of the radiation particle(s), or quanta (the thick line in the figure) were global closed null curves - with the periods of oscillation all equal to the Planck time $T_{l}$ - so the radiation was "frozen" in time $t$. In the macrospace $\mathcal{R}^{3}$, each quantum of the "frozen" radiation covered the distance $L$, equal to its one wavelength, during the time $T_{l}$ which may be regarded as the whole period, or interval of time $t$ which elapsed before the Big Bang. At the moment of the Big Bang, the phase transition $\mathcal{S}^{1} \rightarrow \mathcal{R}^{1}$ of the coordinate time $t$ occurred, which gave the beginning to the expansion of the flat three-dimensional space $\mathcal{R}^{3}$ and to the release of the radiation into the expanding macrospace $\mathcal{R}^{3}$. Note. For reasons of clarity, only the anti-de Sitter two-dimensional spacetime $\mathcal{S}^{1} \times \mathcal{R}^{1}$ is shown, so the figure does not incorporate the flat space $\mathcal{R}^{3}$. Note also that the figure represents the orthogonal projection of the trajectory of a single radiation particle - moving in the spacetime $\mathcal{S}^{1} \times \mathcal{R}^{1} \times \mathcal{R}^{3}$, in a one direction of the macrospace $\mathcal{R}^{3}$ - onto the spacetime $\mathcal{S}^{1} \times \mathcal{R}^{1}$. 